九州大学学術情報リポジトリ

Kyushu University Institutional Repository

\title{
AXIOM OF CHOICE AND ZORN'S LEMMA IN CANTOR CATEGORIES
}

Furusawa, Hitoshi

Department of Mathematics and Computer Science, Kagoshima University

Kawahara, Yasuo

Kyushu University : Professor Emeritus

Tsumagari, Norihiro

Center for Education and Innovation, Sojo University

https://doi.org/10.5109/2232319

出版情報: Bulletin of informatics and cybernetics. 49, pp.11-34, 2017-12. Research Association of Statistical Sciences

バージョン :

権利関係 : 


\section{AXIOM OF CHOICE AND ZORN'S LEMMA IN CANTOR CATEGORIES}

by

Hitoshi Furusawa, Yasuo Kawahara and Norihiro Tsumagari

Reprinted from the Bulletin of Informatics and Cybernetics Research Association of Statistical Sciences, Vol.49

FUKUOKA, JAPAN

2017 


\title{
AXIOM OF CHOICE AND ZORN'S LEMMA IN CANTOR CATEGORIES
}

\author{
By \\ Hitoshi Furusawa* Yasuo Kawahara $^{\dagger}$ and Norihiro TsumagaRI $^{\ddagger}$
}

\begin{abstract}
This paper relationally formulates the axiom of choice and Zorn's lemma. Based on the formalisation, well-known equivalence between the axiom of choice and Zorn's lemma is proved in a manner of relational calculus.
\end{abstract}

Key Words and Phrases: Dedekind categories, Cantor categories, fixpoint theorem, axiom of choice, Zorn's lemma

\section{Introduction}

The axiom of choice in set theory was formulated by E. Zermelo [Zermelo (1904)]. Zorn's lemma [Zorn (1935)] in order theory is known as one of the most important equivalents [Rubin and Rubin (1963)] of the axiom of choice. This paper aims to give a setting and proof for the equivalence between them in Cantor categories.

The calculus of (binary) relations was studied by G. Boole, A. De Morgan, C.S. Peirce and Schröder in 19th century in order to develop a framework for calculational reasoning about theory of relations. A. Tarski initiated the modern investigation of theory of homogenous relations, as relation algebras [Tarski (1941)]. After that, J. Olivier and D. Serrato, and P. Freyd and A. Scedrov developed Dedekind categories [Olivier and Serrato (1980)] and allegories [Freyd and Scedrov (1990)], respectively, as category theory [Mac Lane (1971)] of heterogenous relations. Since the 1970s these algebraic structures have widely been applied as a conceptual and methodological base in areas such as automata theory, graph theory, theory of fuzzy relations, data bases, and semantics of programming languages. Examples and references can be found e.g., in an excellent text book [Schmidt and Ströhlein (1993)] due to G. Schmidt and T. Ströhlein, and the proceedings of the conference series Relational and Algebraic Methods in Computer Science.

Calculational reasonings in the algebraic structure concerning relations are rather formal and allow the use of interactive or automatic theorem provers [Kahl (2014), Pous (2012), Struth and Weber (2014), Höfner and Struth (2008)]. Also manipulating systems for concrete relations [Berghammer and Schmidt (1991), Killingbeck (2015)] are available. Relational studies on well-known fundamental topics in mathematics like this paper contributes for further development of such mechanised mathematical tools.

The paper organises as follows. In Section 2 we define Dedekind categories, which serve naive framework for relational study, and basic notions in Dedekind categories, like

\footnotetext{
* Department of Mathematics and Computer Science, Kagoshima University

$\dagger$ Professor Emeritus, Kyushu University

$¥$ Center for Education and Innovation, Sojo University
} 
univalency and totality of relations, (total) functions and domain relations. With these relational notions three variants $\left(\mathrm{AC}_{*}\right),\left(\mathrm{AC}_{\circ}\right)$ and $(\mathrm{AC}-I)$ of the axiom of choice will be easily introduced. Also, maximum and supremum relations defined by using residual compositions, simply yield a few concepts needed to state Zorn's lemma. However, our equivalence proof of the paper needs more algebraic mechanisms. In Section 3 we give a relational proof for fixpoint theorem [Dugundji (1996)] concerning towers under an additional assumption that every relation in a Dedekind category has its complement. In Section 4 we introduce a concept of Cantor categories, which generalise Dedekind categories so that every relation has rational representation (tabulation), and every object has a membership relation (or, equivalent to the existence of power objects). Also we recall the fundamental propositions in Cantor categories needed in later sections. In Section 5 Zorn's lemma (ZL) will be derived from the axiom of choice $\left(\mathrm{AC}_{*}\right)$ by applying the fixpoint theorem proved in Section 3. We will also prove the axiom of quasi-choice $\left(\mathrm{AC}_{\circ}\right)$ from Zorn's lemma $(\mathrm{ZL})$. In Section 6, as an application of $(\mathrm{ZL})$, a relational proof of Kuratowski's lemma for cliques of relations will be given.

\section{Setup in Dedekind categories}

In this section we reformulate the axiom of choice and Zorn's Lemma with using terminologies in Dedekind categories introduced in [Olivier and Serrato (1980)].

We begin with recalling the definition of Dedekind categories which is the most fundamental structure for this work. Note that Dedekind categories are equivalent to locally complete division allegories introduced in [Freyd and Scedrov (1990)].

A morphism $\alpha$ from an object $X$ into an object $Y$ in a Dedekind category (which will be defined below) is denoted by a half arrow $\alpha: X \rightarrow Y$, and the composite of a morphism $\alpha: X \rightarrow Y$ followed by a morphism $\beta: Y \rightarrow Z$ will be written as $\alpha \beta: X \rightarrow Z$. Also we will denote the identity morphism on $X$ as $\operatorname{id}_{X}$.

Definition 2.1. A Dedekind category $\mathcal{D}$ is a category [Mac Lane (1971)] satisfying the following conditions.

DC1. [Complete Heyting Algebra] For all objects $X$ and $Y$ the hom-set $\mathcal{D}(X, Y)$ consisting of all morphisms of $X$ into $Y$ forms a complete Heyting algebra (equivalent to a complete distributive lattice) with the least morphism $0_{X Y}$ and the greatest morphism $\nabla_{X Y}$. Its algebraic structure will be denoted by

$$
\mathcal{D}(X, Y)=\left(\mathcal{D}(X, Y), \sqsubseteq, \sqcap, \sqcup, \Rightarrow, 0_{X Y}, \nabla_{X Y}\right) .
$$

DC2. [Converse] For all morphisms $\alpha: X \rightarrow Y$ there is a morphism $\alpha^{\sharp}: Y \rightarrow X$, called the converse of $\alpha$, such that for all morphisms $\alpha, \alpha^{\prime}: X \rightarrow Y$ and $\beta: Y \rightarrow Z$ (a) $(\alpha \beta)^{\sharp}=\beta^{\sharp} \alpha^{\sharp}$, (b) $\left(\alpha^{\sharp}\right)^{\sharp}=\alpha$, and (c) $\alpha \sqsubseteq \alpha^{\prime}$ implies $\alpha^{\sharp} \sqsubseteq \alpha^{\prime \sharp}$.

DC3. [Dedekind Formula] For all morphisms $\alpha: X \rightarrow Y, \beta: Y \rightarrow Z$ and $\delta: X \rightarrow Z$ the Dedekind formula (DF for short) $\alpha \beta \sqcap \delta \sqsubseteq \alpha\left(\beta \sqcap \alpha^{\sharp} \delta\right)$ holds.

DC4. [Residual Composition] For all morphisms $\alpha: X \rightarrow Y$ and $\beta: Y \rightarrow Z$ there exists a morphism $\alpha \triangleright \beta: X \rightarrow Z$, called the residual composite of $\alpha$ and $\beta$, such that $\delta \sqsubseteq \alpha \triangleright \beta$ if and only if $\alpha^{\sharp} \delta \sqsubseteq \beta$ for all morphisms $\delta: X \rightarrow Z$.

The following example shows that the notion of Dedekind categories is a framework for theory of relations. 
EXAMPLE 2.2. The category $R e l$ of sets and (binary) relations and the category $\operatorname{Rel}(L)$ of sets and $L$-valued relations [Goguen (1967)] (where $L$ denotes a complete Heyting algebra) form Dedekind categories. More precisely, in Rel each hom-set is a complete Heyting algebra ordered by inclusion and for relations $\alpha: X \rightarrow Y$ and $\beta: Y \rightarrow Z$

- $(y, x) \in \alpha^{\sharp}$ if and only if $(x, y) \in \alpha$,

- $(x, z) \in \alpha \beta$ if and only if there exists $y \in Y$ such that $(x, y) \in \alpha$ and $(y, z) \in \beta$,

- $(x, z) \in \alpha \triangleright \beta$ if and only if $(x, y) \in \alpha$ implies $(y, z) \in \beta$ for all $y \in Y$.

In what follows, the word relation is a synonym for morphism of a Dedekind category. A relation $\alpha: X \rightarrow Y$ in a Dedekind category is univalent if $\alpha^{\sharp} \alpha \sqsubseteq \mathrm{id}_{Y}$, and it is total if $\operatorname{id}_{X} \sqsubseteq \alpha \alpha^{\sharp}$. A univalent and total relation is called a function (tfn, for short) and may be introduced as $f: X \rightarrow Y$. An injection $i: X \rightarrow Y$ is a function such that $i i^{\sharp}=\mathrm{id}_{X}$. The domain relation $\lfloor\alpha\rfloor: X \rightarrow X$ of a relation $\alpha: X \rightarrow Y$ is defined as $\lfloor\alpha\rfloor=\alpha \alpha^{\sharp} \sqcap \operatorname{id}_{X}$. A relation $\alpha$ is total iff $\lfloor\alpha\rfloor=\operatorname{id}_{X}$.

The following proposition lists a part of the basic properties of Dedekind categories without proof.

Proposition 2.3. Let $\alpha, \alpha^{\prime}: X \rightarrow Y, \beta, \beta^{\prime}: Y \rightarrow Z, \gamma: Z \rightarrow W, \delta: U \rightarrow Z$, $\zeta: X \rightarrow Z$ and $\eta: Y \rightarrow W$ be relations in a Dedekind category. Then the following holds.

(a) $\alpha 0_{Y Z}=0_{X Z}$ and $0_{V X} \alpha=0_{V Y}$.

(b) If $\alpha \sqsubseteq \alpha^{\prime}$ and $\beta \sqsubseteq \beta^{\prime}$ then $\alpha \beta \sqsubseteq \alpha^{\prime} \beta^{\prime}$ and $\alpha^{\prime} \triangleright \beta \sqsubseteq \alpha \triangleright \beta^{\prime}$,

(c) $\alpha\left(\sqcup_{\lambda \in \Lambda} \beta_{\lambda}\right) \gamma=\sqcup_{\lambda \in \Lambda} \alpha \beta_{\lambda} \gamma$ and $\alpha\left(\sqcap_{\lambda \in \Lambda} \beta_{\lambda}\right) \gamma \sqsubseteq \sqcap_{\lambda \in \Lambda} \alpha \beta_{\lambda} \gamma$.

(d) If $\alpha$ and $\delta$ are univalent then $\alpha\left(\beta \sqcap \beta^{\prime}\right) \delta^{\sharp}=\alpha \beta \delta^{\sharp} \sqcap \alpha \beta^{\prime} \delta^{\sharp}$,

(e) $\alpha=\lfloor\alpha\rfloor \alpha$,

(f) If $\alpha \sqsubseteq \alpha^{\prime},\lfloor\alpha\rfloor=\left\lfloor\alpha^{\prime}\right\rfloor$ and $\alpha^{\prime}$ is univalent, then $\alpha=\alpha^{\prime}$.

(g) $\lfloor\alpha\rfloor \sqsubseteq\left\lfloor\zeta\right.$ iff $\alpha \nabla_{Y X} \sqsubseteq \zeta \nabla_{Z X}$.

(h) If $\alpha$ and $\alpha^{\prime}$ are univalent and $\lfloor\alpha\rfloor \sqcap\left\lfloor\alpha^{\prime}\right\rfloor=0_{X Y}$, then $\alpha \sqcup \alpha^{\prime}$ is also univalent,

(i) $\alpha \triangleright(\beta \triangleright \gamma)=\alpha \beta \triangleright \gamma$ and $(\alpha \triangleright \beta) \gamma \sqsubseteq \alpha \triangleright \beta \gamma$,

(j) $\left(\alpha \sqcup \alpha^{\prime}\right) \triangleright \beta=(\alpha \triangleright \beta) \sqcap\left(\alpha^{\prime} \triangleright \beta\right)$ and $\alpha \triangleright\left(\beta \sqcap \beta^{\prime}\right)=(\alpha \triangleright \beta) \sqcap\left(\alpha \triangleright \beta^{\prime}\right)$,

(k) $(\alpha \triangleright \beta)\left(\beta^{\sharp} \triangleright \eta\right) \sqsubseteq \alpha \triangleright \eta$,

(l) $\alpha \sqsubseteq(\alpha \triangleright \beta) \triangleright \beta^{\sharp}$,

(m) $\alpha \triangleright \beta=\left((\alpha \triangleright \beta) \triangleright \beta^{\sharp}\right) \triangleright \beta$,

(n) If $\alpha$ is a function then $\alpha \triangleright \beta=\alpha \beta$ and $\alpha(\beta \triangleright \gamma)=\alpha \beta \triangleright \gamma$,

(o) If $\beta$ is a function then $\alpha \beta \triangleright \gamma=\alpha \triangleright \beta \gamma$, 
(p) If $\delta$ is a function then $(\alpha \triangleright \beta) \delta^{\sharp}=\alpha \triangleright \beta \delta^{\sharp}$.

In a Dedekind category, for an injection $i: S \rightarrow Y$, a function $f: X \rightarrow Y$ satisfying $f f^{\sharp} \sqsubseteq i i^{\sharp}$ bijectively corresponds to a function $h: X \rightarrow S: f=h i$ if and only if $h=f i i^{\sharp}$. This bijective correspondence is called the injection adjunction and denoted by the following notation.

$$
\frac{f: X \rightarrow Y \quad\left\{f^{\sharp} f \sqsubseteq i^{\sharp} i\right\}}{h: X \rightarrow S} \quad \frac{f=h i}{h=f i^{\sharp}} \quad \text { (injection adjunction) }
$$

In addition, an object $I$ in a Dedekind category $\mathcal{D}$ is called unit if it satisfies $0_{I I} \neq \mathrm{id}_{I}=$ $\nabla_{I I}$ and $\nabla_{X I} \nabla_{I X}=\nabla_{X X}$ for all objects $X$.

Example 2.4. Consider the Dedekind category Rel.

- A function $f: X \rightarrow Y$ corresponds to the function $h: X \rightarrow S$ which maps $x \in X$ to $f(x) \in S$ by the injection adjunction for any sets $S$ such that $\{f(x): x \in X\} \subseteq$ $S \subseteq Y$.

- A singleton set is the unit.

In the rest of this section, we assume that a Dedekind category has a unit without explicit mentioning if its existence is necessary.

Now define some auxiliary notions concerned with axiom of choice in Dedekind categories. Let $\alpha: X \rightarrow Y$ be a relation. A univalent relation $\gamma: X \rightarrow Y$ is called a choice of $\alpha$ if $\gamma \sqsubseteq \alpha$ and $\lfloor\gamma\rfloor=\lfloor\alpha\rfloor$. A choice of a total relation is a function. Also we call a univalent relation $\gamma: X \neg Y$ a quasi-choice of $\alpha$ if $\gamma$ is maximal among all univalent relations contained in $\alpha$, that is, (i) $\gamma \sqsubseteq \alpha$, and (ii) $\gamma \sqsubseteq \delta \sqsubseteq \alpha$ and $\delta^{\sharp} \delta \sqsubseteq \operatorname{id}_{Y}$ imply $\gamma=\delta$. A choice of $\alpha$ is a quasi-choice of $\alpha$.

With these terminologies we may formulate the axiom of choice $\left(\mathrm{AC}_{*}\right)$, the axiom of quasi-choice $\left(\mathrm{AC}_{\circ}\right)$ and the restricted axiom of choice $(\mathrm{AC}-\mathrm{I})$ in Dedekind categories as follows.

Definition 2.5. $\left(\mathrm{AC}_{*}\right)$ Every relation $\alpha: X \rightarrow Y$ has a choice.

$\left(\mathrm{AC}_{\circ}\right)$ Every relation $\alpha: X \rightarrow Y$ has a quasi-choice.

(AC- $I$ ) Every relation $\rho: I \rightarrow X$ has a choice.

Remark that $\left(\mathrm{NE}_{*}\right) \leftrightarrow\left(\operatorname{Tot}_{*}\right) \wedge(\mathrm{AC}-I)$, where $\left(\mathrm{Tot}_{*}\right)$ and $\left(\mathrm{NE}_{*}\right)$ are the axiom of totality and the axiom of nonemptyness, namely,

$\left(\operatorname{Tot}_{*}\right)$ every nonzero relation $\rho: I \rightarrow X$ is total,

$\left(\mathrm{NE}_{*}\right)$ every nonzero relation $\rho: I \rightarrow X$ includes some function $x: I \rightarrow X$

discussed in [Furusawa and Kawahara (2015)], respectively.

Example 2.6. - The axiom of choice $\left(\mathrm{AC}_{*}\right)$ in the Dedekind category $R e l$ is a variant appeared as property AC4 in [Rubin and Rubin (1963)] of the axiom of choice in set theory. The restricted axiom of choice (AC- $I$ ) on the unit $I$ clearly argues a trivial fact that we may choose an element of a nonempty set, and it is usually omitted in set-theoretical argument. 
- In the Dedekind category $\operatorname{Rel}(L)$, equivalences $\left(\mathrm{NE}_{*}\right) \leftrightarrow\left(\operatorname{Tot}_{*}\right) \leftrightarrow L=\{0,1\}$ hold. Also $(\mathrm{AC}-I)$ holds in $\operatorname{Rel}(L)$ if $L$ is a finite linearly ordered set. Moreover it is proved in [Furusawa and Kawahara (2015)] that If $L$ is a complete Boolean algebra, $\operatorname{Rel}(L)$ satisfies $\left(\mathrm{AC}_{*}\right)$.

For a relation $\xi: X \rightarrow X$ the maximum relation $\max (\rho, \xi): V \rightarrow X$ and supremum relation $\sup (\rho, \xi): V \rightarrow X$ of a relation $\rho: V \rightarrow X$ (with respect to $\xi$ ) are defined as follows.

$$
\max (\rho, \xi)=\rho \sqcap(\rho \triangleright \xi) \text { and } \sup (\rho, \xi)=(\rho \triangleright \xi) \sqcap\left((\rho \triangleright \xi) \triangleright \xi^{\sharp}\right) \text {. }
$$

The following proposition lists some of the basic properties of maximum relations and supremum relations.

Proposition 2.7. Let $\rho: V \rightarrow X$ and $\xi: X \neg X$ be relations in a Dedekind category. Then the following holds.

(a) $\sup \left(\rho, \xi^{\sharp}\right)=\sup \left(\rho \triangleright \xi^{\sharp}, \xi\right)$,

(b) $\sup (\rho, \xi) \sqsubseteq \rho \triangleright \xi \sqsubseteq \sup (\rho, \xi) \triangleright \xi$,

(c) If $\xi \xi \sqsubseteq \xi$ and $\sup (\rho, \xi)$ is total then $\sup (\rho, \xi) \xi=\rho \triangleright \xi$,

(d) If $f: W \rightarrow V$ is a function then $f \sup (\rho, \xi)=\sup (f \rho, \xi)$,

(e) If $\xi \sqcap \xi^{\sharp} \sqsubseteq \operatorname{id}_{X}$ then $\max (\rho, \xi)$ and $\sup (\rho, \xi)$ are univalent.

(f) If $\sigma: V \rightarrow C$ is a relation and $i: C \rightarrow X$ is a function then $\sup (\sigma i, \xi) i^{\sharp} \sqsubseteq$ $\sup \left(\sigma, i \xi i^{\sharp}\right)$.

To state Zorn's lemma in Dedekind categories we need some basic notions related to orders.

Definition 2.8. Let $\xi: X \rightarrow X$ be a relation and $V$ an object.

(a) A relation $\rho: V \rightarrow X$ is a $\xi$-chain if $\rho^{\sharp} \rho \sqsubseteq \xi \sqcup \xi^{\sharp}$.

(b) $\xi$ is complete on $V$ if $\sup (\rho, \xi)$ is total for all relations $\rho: V \rightarrow X$.

(c) $\xi$ is chain complete on $V$ if $\sup (\rho, \xi)$ is total for all $\xi$-chains $\rho: V \rightarrow X$.

(d) $\xi$ is inductive on $V$ if $\rho \triangleright \xi$ is total for all $\xi$-chains $\rho: V \rightarrow X$.

(e) A function $x: V \rightarrow X$ is $\xi$-maximal if $x \xi \sqsubseteq x$.

Obviously a complete relation is chain complete and a chain complete relation is inductive. A relation $\xi: X \rightarrow X$ in a Dedekind category is called a (partial) order if it is reflexive $\left(\operatorname{id}_{X} \sqsubseteq \xi\right)$, transitive $(\xi \xi \sqsubseteq \xi)$ and antisymmetric $\left(\xi \sqcap \xi^{\sharp} \sqsubseteq \mathrm{id}_{X}\right)$. Note that $\xi^{\sharp} \triangleright \xi=\xi$ iff $\operatorname{id}_{X} \sqsubseteq \xi$ and $\xi \xi \sqsubseteq \xi$. For a reflexive relation $\xi: X \neg X$, a function $x: V \rightarrow X$ is $\xi$-maximal iff $x \xi=x$. 
Example 2.9. Consider the Dedekind category Rel. Since the unit $I$ in $R e l$ is a singleton set, a relation and a function from $I$ to $X$ may be identified with a subset and an element of $X$, respectively. An order $\xi: X \rightarrow X$ in Rel is a partial order on the set $X$ of course. Thus the residual composite $\rho \triangleright \xi$ of a relation $\rho: I \neg X$ and an order $\xi: X \neg X$ may be identified with the set of upper bounds of $\rho$ with respect to the order $\xi$. Moreover, for a relation $\rho: I \rightarrow X$ and an order $\xi: X \rightarrow X$,

- $\max (\rho, \xi)$ and $\sup (\rho, \xi)$ respectively correspond to maximum and supremum elements of $\rho$ with respect to $\xi$ if they exist,

- $\xi$-chain $\rho$ is a totally ordered subset of $X$ with respect to $\xi$,

- totality of $\sup (\rho, \xi)$ and $\rho \triangleright \xi$ respectively represents existence of supremum and upper bounds of $\rho$ with respect to $\xi$, and

- $\xi$-maximal function $x: I \rightarrow X$ corresponds to a maximal element of $X$ with respect to $\xi$.

With these terminologies Zorn's lemma (ZL) in Dedekind categories is stated as follows.

Definition 2.10. (ZL) For all inductive orders $\xi: X \rightarrow X$ on $V$ there exists a $\xi$-maximal function $x: V \rightarrow X$.

As our equivalence proof of axiom of choice and Zorn's lemma requires more algebraic mechanisms, three additional conditions will be imposed on Dedekind categories in Section 3 and 4 .

\section{Fixpoint Theorem}

In this section we will show a fixpoint theorem which is helpful for the proof that (AC) implies (ZL). Our proof of (ZL) from (AC) follows a proof in [Dugundji (1996)] using a notion of towers and a fixpoint theorem related to them.

The following notion plays a part of key rôles in the fixpoint theorem.

Definition 3.1. For a relation $\xi: X \rightarrow X$ a function $g: X \rightarrow X$ is called a $\xi$-section if $g \sqsubseteq \xi$ and $\xi \sqcap g \xi^{\sharp} \sqsubseteq \operatorname{id}_{X} \sqcup g$.

The next example provides a set theoretical intuition of $\xi$-sections.

ExAmple 3.2. In the Dedekind category Rel, for an order $\xi$ on a set $X$ a $\xi$-section maps $x \in X$ either to $x$ itself or to a cover of $x$ with respect to $\xi$. More precisely, we intend the notion of a $\xi$-section to be the endomap on the power set of a partially ordered set which assigns the set $S \cup\left\{s_{0}\right\}$ to a set $S$, where $s_{0}$ is the supremum of $S$.

We show a basic property of $\xi$-sections needed later.

Proposition 3.3. Let $\xi: X \rightarrow X$ be a reflexive relation. If $g: X \rightarrow X$ is a $\xi$-section, then the inclusion $\left(\xi \sqcup \xi^{\sharp}\right) \sqcap\left(g \xi \sqcup \xi^{\sharp} g\right) \sqsubseteq g \xi \sqcup \xi^{\sharp}$ holds. 
ProOF. It is direct from

$$
\begin{array}{rlll}
\left(\xi \sqcup \xi^{\sharp}\right) \sqcap\left(\xi^{\sharp} g \sqcup g \xi\right) & \sqsubseteq & \left(\xi \sqcap \xi^{\sharp} g\right) \sqcup g \xi \sqcup \xi^{\sharp} & \\
& \sqsubseteq\left(\xi g^{\sharp} \sqcap \xi^{\sharp}\right) g \sqcup g \xi \sqcup \xi^{\sharp} & & \{\mathrm{DF}\} \\
& \sqsubseteq\left(\mathrm{id}_{X} \sqcup g^{\sharp}\right) g \sqcup g \xi \sqcup \xi^{\sharp} & \left\{\xi \sqcap g \xi^{\sharp} \sqsubseteq \mathrm{id}_{X} \sqcup g\right\} \\
& \sqsubseteq g \sqcup \mathrm{id}_{X} \sqcup g \xi \sqcup \xi^{\sharp} & & \left\{g^{\sharp} g \sqsubseteq \mathrm{id}_{X}\right\} \\
& =g \xi \sqcup \xi^{\sharp} . & & \left\{\operatorname{id}_{X} \sqsubseteq \xi\right\}
\end{array}
$$

Let $\alpha: X \rightarrow Y$ be a relation in a Dedekind category. We call a relation $\alpha^{-}: X \rightarrow Y$ satisfying $\alpha \sqcup \alpha^{-}=\nabla_{X Y}$ and $\alpha \sqcap \alpha^{-}=0_{X Y}$ a complement of $\alpha$. The complement $\alpha^{-}$ is unique if it exists, and the identity $\alpha^{-}=\alpha \Rightarrow 0_{X Y}$ holds.

The axiom of complements $(\mathrm{Ba})$ for a Dedekind category is the following condition.

(Ba) Every relation $\alpha: X \rightarrow Y$ has a complement.

This means that the internal structure of Dedekind categories satisfying (Ba) obeys the classical logic in a sense that each hom-set forms a complete boolean algebra. Note that Dedekind categories satisfying (Ba) are equivalent to Schröder categories introduced in [Olivier and Serrato (1980)].

Now we review some basic properties of residual compositions in Schröder categories.

Lemma 3.4. Let $\alpha: X \rightarrow Y, \beta, \beta^{\prime}: Y \rightarrow Z, \gamma: X \rightarrow Z$ and $\delta: W \rightarrow Z$ be relations in a Dedekind category satisfying (Ba). Then the following holds.

(a) $\alpha \beta \sqsubseteq \gamma \leftrightarrow \alpha^{\sharp} \gamma^{-} \sqsubseteq \beta^{-} \leftrightarrow \gamma^{-} \beta^{\sharp} \sqsubseteq \alpha^{-}$,

(Schröder equivalence)

(b) $\gamma \sqsubseteq \alpha \triangleright \beta \leftrightarrow \alpha \sqsubseteq \gamma \triangleright \beta^{\sharp}$,

(Galois connection)

(c) $\alpha \triangleright \beta=\left(\alpha \beta^{-}\right)^{-}$,

(d) $(\alpha \triangleright \beta)^{\sharp}=\beta^{-\sharp} \triangleright \alpha^{-\sharp}$,

(e) If $\alpha$ and $\delta$ are functions then $\left(\alpha \beta \delta^{\sharp}\right)^{-}=\alpha \beta^{-} \delta^{\sharp}$,

(f) $\alpha \triangleright\left(\beta \sqcup \beta^{\prime}\right) \sqsubseteq \alpha \beta \sqcup\left(\alpha \triangleright \beta^{\prime}\right)$.

Proof. (a) First we will prove the implication $\alpha \beta \sqsubseteq \gamma \rightarrow \alpha^{\sharp} \gamma^{-} \sqsubseteq \beta^{-}$. Assume $\alpha \beta \sqsubseteq \gamma$, which is equivalent to $\alpha \beta \sqcap \gamma^{-}=0_{X Z}$. Then

$$
\begin{aligned}
\alpha^{\sharp} \gamma^{-} \sqcap \beta & \sqsubseteq \alpha^{\sharp}\left(\gamma^{-} \sqcap \alpha \beta\right) & & \{\mathrm{DF}\} \\
& =\alpha^{\sharp} 0_{X Z} & & \left\{\alpha \beta \sqcap \gamma^{-}=0_{X Z}\right\} \\
& =0_{Y Z}, & & \text { zero law }\}
\end{aligned}
$$

which implies $\alpha^{\sharp} \gamma^{-} \sqsubseteq \beta^{-}$. The converse implication $\alpha^{\sharp} \gamma^{-} \sqsubseteq \beta^{-} \rightarrow \alpha \beta \sqsubseteq \gamma$ is a variant of the first implication. The proof of another equivalence $\alpha \beta \sqsubseteq \gamma \leftrightarrow \gamma^{-} \beta^{\sharp} \sqsubseteq \alpha^{-}$is analogous.

(b) The Galois connection is clear from

$$
\begin{aligned}
\gamma \sqsubseteq \alpha \triangleright \beta & \leftrightarrow \alpha^{\sharp} \gamma \sqsubseteq \beta & & \text { residual equiv. }\} \\
& \leftrightarrow \gamma^{\sharp} \alpha \sqsubseteq \beta^{\sharp} & & \text { converse \} } \\
& \leftrightarrow \alpha \sqsubseteq \gamma \triangleright \beta^{\sharp} . & & \text { residual equiv. }\}
\end{aligned}
$$


(c) $\alpha \triangleright \beta=\left(\alpha \beta^{-}\right)^{-}$follows from

$$
\begin{aligned}
\gamma \sqsubseteq \alpha \triangleright \beta & \leftrightarrow \alpha^{\sharp} \gamma \sqsubseteq \beta & & \{\text { residual equiv. }\} \\
& \leftrightarrow \alpha \beta^{-} \sqsubseteq \gamma^{-} & & \{\text {(a) Schröder }\} \\
& \leftrightarrow \gamma \sqsubseteq\left(\alpha \beta^{-}\right)^{-} . & & \{\text {complement }\}
\end{aligned}
$$

(d) $(\alpha \triangleright \beta)^{\sharp}=\beta^{-\sharp} \triangleright \alpha^{-\sharp}$ holds by

$$
\begin{aligned}
& (\alpha \triangleright \beta)^{\sharp}=\left(\alpha \beta^{-}\right)^{-\sharp} \quad\{(\mathrm{c})\} \\
& =\left(\beta^{-\sharp} \alpha^{\sharp}\right)^{-} \quad\left\{\beta^{-\sharp}=\beta^{\sharp-}\right\} \\
& =\left(\beta^{-\sharp} \alpha^{-\sharp-}\right)^{-} \quad\left\{\alpha^{--}=\alpha\right\} \\
& =\beta^{-\sharp} \triangleright \alpha^{-\sharp} . \quad\{(\mathrm{c})\}
\end{aligned}
$$

(e) If $\alpha$ and $\delta$ are functions, then

$$
\begin{array}{rlll}
\nabla_{X W} & \sqsubseteq \alpha \alpha^{\sharp} \nabla_{X W} \delta \delta^{\sharp} & & \{\alpha, \delta: \text { total }\} \\
& \sqsubseteq \alpha \nabla_{Y Z} \delta^{\sharp} & & \left\{\alpha^{\sharp} \nabla_{X W} \delta \sqsubseteq \nabla_{Y Z}\right\} \\
& =\alpha\left(\beta \sqcup \beta^{-}\right) \delta^{\sharp} & \left\{\beta \sqcup \beta^{-}=\nabla_{Y Z}\right\} \\
& =\alpha \beta \delta^{\sharp} \sqcup \alpha \beta^{-} \delta^{\sharp} &
\end{array}
$$

and

$$
\begin{aligned}
\alpha \beta \delta^{\sharp} \sqcap \alpha \beta^{-} \delta^{\sharp} & \sqsubseteq \alpha\left(\beta \sqcap \alpha^{\sharp} \alpha \beta^{-} \delta^{\sharp} \delta\right) \delta^{\sharp} & & \{\mathrm{DF}\} \\
& \sqsubseteq \alpha\left(\beta \sqcap \beta^{-}\right) \delta^{\sharp} & & \{\alpha, \beta \text { : univalent }\} \\
& =0_{X W} & & \left\{\beta \sqcap \beta^{-}=0_{Y Z}\right\}
\end{aligned}
$$

hold. Hence $\alpha \beta^{-} \delta^{\sharp}=\left(\alpha \beta \delta^{\sharp}\right)^{-}$by the uniqueness of complements.

(f) $\alpha \triangleright\left(\beta \sqcup \beta^{\prime}\right) \sqsubseteq \alpha \beta \sqcup\left(\alpha \triangleright \beta^{\prime}\right)$ is equivalent to $\left(\alpha \triangleright\left(\beta \sqcup \beta^{\prime}\right)\right) \sqcap(\alpha \beta)^{-} \sqsubseteq \alpha \triangleright \beta^{\prime}$ by the Horn rule and the latter follows from

$$
\begin{array}{rlr} 
& \left(\alpha \triangleright\left(\beta \sqcup \beta^{\prime}\right)\right) \sqcap(\alpha \beta)^{-} & \\
=\quad\left(\alpha \triangleright\left(\beta \sqcup \beta^{\prime}\right)\right) \sqcap\left(\alpha \triangleright \beta^{-}\right) & \{(\mathrm{c})\} \\
=\alpha \triangleright\left(\left(\beta \sqcup \beta^{\prime}\right) \sqcap \beta^{-}\right) & \{2.3(\mathrm{j})\} \\
\sqsubseteq \alpha \triangleright \beta^{\prime} . & \left\{\left(\beta \sqcup \beta^{\prime}\right) \sqcap \beta^{-} \sqsubseteq \beta^{\prime}\right\}
\end{array}
$$

In a Schröder category, letting $\xi_{\circ}=\xi \sqcap \mathrm{id}_{X}^{-}$, a function $g: X \rightarrow X$ is a $\xi$-section if and only if $g \sqcap \xi_{\circ} \xi_{\circ}=0_{X X}$ by

$$
\begin{aligned}
\xi \sqcap g \xi^{\sharp} \sqsubseteq \operatorname{id}_{X} \sqcup g & \leftrightarrow g \xi^{\sharp} \sqcap g^{-} \sqsubseteq \xi^{-} \sqcup \operatorname{id}_{X} & & \{\text { Horn rule }\} \\
& \leftrightarrow g\left(\xi^{\sharp} \sqcap \operatorname{id}_{X}^{-}\right) \sqsubseteq\left(\xi \sqcap \operatorname{id}_{X}^{-}\right)^{-} & & \left\{g^{-}=g \operatorname{id}_{X}^{-}\right\} \\
& \leftrightarrow g \xi_{\circ}^{\sharp} \sqsubseteq \xi_{\circ}^{-} & & \left\{\xi_{\circ}=\xi \sqcap \operatorname{id}_{X}^{-}\right\} \\
& \leftrightarrow \xi_{\circ} \xi_{\circ} \sqsubseteq g^{-} & & \{\text {Schröder equiv. }\} \\
& \leftrightarrow g \sqcap \xi_{\circ} \xi_{\circ}=0_{X X} . & & \{\text { complement }\}
\end{aligned}
$$

In addition, the following two properties hold in any Schröder categories.

Proposition 3.5. Let $\xi: X \rightarrow X$ be an order, $g: X \rightarrow X$ a function, and $\sigma, \mu: V \rightarrow X$ relations. If $\sigma \sqsubseteq \mu \triangleright\left(g \xi \sqcup \xi^{\sharp}\right)$, then $\sup (\sigma, \xi) \sqsubseteq \mu \triangleright\left(g \xi \sqcup \xi^{\sharp}\right)$. 
Proof. (1) $\sigma \triangleright \xi \sqsubseteq \sigma \xi^{\sharp} g^{\sharp} \triangleright g \xi$ and (2) $\mu \sqsubseteq \sigma \xi^{\sharp} g^{\sharp} \sqcup(\sigma \triangleright \xi)$ hold by

$$
\begin{aligned}
\sigma \triangleright \xi & =\sigma \xi^{\sharp} \triangleright \xi & & \left\{\xi=\xi^{\sharp} \triangleright \xi\right\} \\
& \sqsubseteq \sigma \xi^{\sharp} g^{\sharp} g \triangleright \xi & & \left\{g^{\sharp} g \sqsubseteq \mathrm{id}_{X}\right\} \\
& =\sigma \xi^{\sharp} g^{\sharp} \triangleright g \xi . & & \{g: \text { function }\}
\end{aligned}
$$

and

$$
\begin{array}{rll}
\mu & \sqsubseteq \sigma \triangleright\left(\xi^{\sharp} g^{\sharp} \sqcup \xi\right) & \left\{\sigma \sqsubseteq \mu \triangleright\left(g \xi \sqcup \xi^{\sharp}\right)\right\} \\
& \sqsubseteq \sigma \xi^{\sharp} g^{\sharp} \sqcup(\sigma \triangleright \xi) . & \{3.4(\mathrm{f})\}
\end{array}
$$

Thus we have

$$
\begin{aligned}
\sup (\sigma, \xi) & =(\sigma \triangleright \xi) \sqcap\left((\sigma \triangleright \xi) \triangleright \xi^{\sharp}\right) \\
& \sqsubseteq \quad\left(\sigma \xi^{\sharp} g^{\sharp} \triangleright g \xi\right) \sqcap\left((\sigma \triangleright \xi) \triangleright \xi^{\sharp}\right) \\
& \sqsubseteq\left(\sigma \xi^{\sharp} g^{\sharp}\left(g \xi \sqcup \xi^{\sharp}\right)\right) \sqcap\left((\sigma \triangleright \xi) \triangleright\left(g \xi \sqcup \xi^{\sharp}\right)\right) \\
& =\left(\sigma \xi^{\sharp} g^{\sharp} \sqcup(\sigma \triangleright \xi)\right) \triangleright\left(g \xi \sqcup \xi^{\sharp}\right) \\
& \sqsubseteq \mu \triangleright\left(g \xi \sqcup \xi^{\sharp}\right) .
\end{aligned}
$$

Proposition 3.6. Let $\gamma: C \rightarrow X$ be a univalent relation and $\xi: C \rightarrow C$ an order such that $\xi=\varepsilon \triangleright \varepsilon^{\sharp}$ for a relation $\varepsilon: C \rightarrow X$. If a function $g: C \rightarrow C$ satisfies $g \varepsilon=\varepsilon \sqcup \gamma$, then $g$ is a $\xi$-section.

Proof. $g \sqsubseteq \xi$ follows from

$$
\begin{aligned}
g & \sqsubseteq \varepsilon \triangleright \varepsilon^{\sharp} g & & \left\{\varepsilon^{\sharp} g \sqsubseteq \varepsilon^{\sharp} g\right\} \\
& \sqsubseteq \varepsilon \triangleright(g \varepsilon)^{\sharp} g & & \{\varepsilon \sqsubseteq \varepsilon \sqcup \gamma=g \varepsilon\} \\
& \sqsubseteq \varepsilon \triangleright \varepsilon^{\sharp} & & \left\{g^{\sharp} g \sqsubseteq \operatorname{id}_{C}\right\} \\
& =\xi . & & \left\{\varepsilon \triangleright \varepsilon^{\sharp}=\xi\right\}
\end{aligned}
$$

Next (1) $\xi \sqcap \gamma \varepsilon^{\sharp} \sqsubseteq g \xi$, (2) $\xi g^{\sharp} \sqsubseteq \varepsilon \gamma^{\sharp} \sqcup \xi$ and (3) $\xi \sqcap g \xi^{\sharp} \sqsubseteq g \xi \sqcup \operatorname{id}_{C}$ hold by

and

$$
\begin{array}{rlrl}
\xi \sqcap \gamma \varepsilon^{\sharp} & \sqsubseteq \xi \sqcap\left(\gamma \triangleright \varepsilon^{\sharp}\right) & & \left\{\gamma^{\sharp} \gamma \sqsubseteq \mathrm{id}_{X}\right\} \\
& =(\varepsilon \sqcup \gamma) \triangleright \varepsilon^{\sharp} & & \left\{\xi=\varepsilon \triangleright \varepsilon^{\sharp}\right\} \\
& =g \varepsilon \triangleright \varepsilon^{\sharp} & & \{\varepsilon \sqcup \gamma=g \varepsilon\} \\
& =g \xi, & & \left\{g: \mathrm{tfn}, \varepsilon \triangleright \varepsilon^{\sharp}=\xi\right\} \\
\xi g^{\sharp}=\varepsilon \triangleright \varepsilon^{\sharp} g^{\sharp} & & \left\{\xi=\varepsilon \triangleright \varepsilon^{\sharp}, g: \text { tfn }\right\} \\
& =\varepsilon \triangleright\left(\varepsilon^{\sharp} \sqcup \gamma^{\sharp}\right) & & \{g \varepsilon=\varepsilon \sqcup \gamma\} \\
& \sqsubseteq \varepsilon \gamma^{\sharp} \sqcup\left(\varepsilon \triangleright \varepsilon^{\sharp}\right) & & \{3.4(\mathrm{f})\} \\
& =\varepsilon \gamma^{\sharp} \sqcup \xi, & & \left\{\varepsilon \triangleright \varepsilon^{\sharp}=\xi\right\}
\end{array}
$$

and

$$
\begin{array}{rlrl}
\xi \sqcap g \xi^{\sharp} & \sqsubseteq \xi \sqcap\left(\gamma \varepsilon^{\sharp} \sqcup \xi^{\sharp}\right) & \{(2)\} & \\
& =\left(\xi \sqcap \gamma \varepsilon^{\sharp}\right) \sqcup\left(\xi \sqcap \xi^{\sharp}\right) & \\
& \sqsubseteq g \xi \sqcup \operatorname{id}_{C} . & & \{(1), \xi \text { : antisymmetric }\}
\end{array}
$$

Therefore we have

$$
\begin{aligned}
& \xi \sqcap g \xi^{\sharp} \sqsubseteq\left(g \xi \sqcup \operatorname{id}_{C}\right) \sqcap g \xi^{\sharp} \quad\{(3)\} \\
& \sqsubseteq\left(g \xi \sqcap g \xi^{\sharp}\right) \sqcup \mathrm{id}_{C} \\
& =g\left(\xi \sqcap \xi^{\sharp}\right) \sqcup \operatorname{id}_{C} \quad\{g: \text { function }\} \\
& \sqsubseteq g \sqcup \operatorname{id}_{C} . \quad\{\xi: \text { antisymmetric }\}
\end{aligned}
$$


A relational version of the concept of towers [Dugundji (1996)] will be defined in a Dedekind category as follows.

Definition 3.7. Let $\xi: X \rightarrow X$ be a relation and $g: X \rightarrow X$ a function. A relation $\tau: V \rightarrow X$ is called a $(\xi, g)$-tower if it satisfies the following conditions.

$(\mathrm{T} 1) \sup (\sigma, \xi) \sqsubseteq \tau$ for all $\xi$-chains $\sigma: V \rightarrow X$ such that $\sigma \sqsubseteq \tau$,

(T2) $\tau g \sqsubseteq \tau$.

The next example provides a set theoretical intuition of $(\xi, g)$-towers.

ExAmple 3.8. In the Dedekind category Rel, if $V$ is a singleton set and $\xi$ is an order on a set $X$, a $(\xi, g)$-tower $\tau$

- contains the supremum of every $\xi$-chain $\rho$ included by $\tau$ and

- includes image of $\tau$ under $g$,

with identifying $\tau$ with a subset of $X$. Moreover, let $X$ be the power set of a set, $\xi$ the inclusion and $\left.g\right|_{\tau}$ the restriction of $g$ to $\tau$. Then, if $\tau$ is a $(\xi, g)$-tower and contains the empty subset of $X, \tau$ is a $\left.g\right|_{\tau}$-tower in the sense of [Dugundji (1996)].

The universal relation $\nabla_{V X}$ is a $(\xi, g)$-tower and so $(\xi, g)$-towers do exist. It is trivial that the meet of all $(\xi, g)$-towers $\tau: V \rightarrow X$ is also a $(\xi, g)$-tower and is the minimum $(\xi, g)$-tower. Hence for each object $V$ the minimum $(\xi, g)$-tower $\tau_{0}: V \rightarrow X$ exists. The minimum tower will play an important rôle to prove a fixpoint theorem below. Although it is possible to define towers and the minimum tower exists in any Dedekind categories, the next theorem is satisfied by a Schröder category.

Theorem 3.9. Let $\xi: X \rightarrow X$ be an order, $g: X \rightarrow X$ a $\xi$-section and $\tau_{0}: V \rightarrow X$ the minimum $(\xi, g)$-tower. Set $\mu=\tau_{0} \triangleright\left(\xi^{\sharp} \sqcup \xi\right)$ and $\mu^{\prime}=\tau_{0} \sqcap\left(\mu \triangleright\left(g \xi \sqcup \xi^{\sharp}\right)\right)$. Then the following holds.

(a) $\mu^{\prime}$ is a $(\xi, g)$-tower,

(b) $\mu$ is a $(\xi, g)$-tower,

(c) $\tau_{0}$ is a $\xi$-chain,

(d) $\sup \left(\tau_{0}, \xi\right) g \sqsubseteq \max \left(\tau_{0}, \xi\right)$,

(e) If $\xi$ is chain complete on $V$, then $\sup \left(\tau_{0}, \xi\right) g=\sup \left(\tau_{0}, \xi\right)$.

Proof. (a) (T1) Let $\sigma: V \rightarrow X$ be a $\xi$-chain such that $\sigma \sqsubseteq \mu^{\prime}$. Then it is clear that $\sigma \sqsubseteq \mu^{\prime} \sqsubseteq \tau_{0}$ and $\sup (\sigma, \xi) \sqsubseteq \tau_{0}$ by the condition (T1) for $\tau_{0}$. On the other hand, since $\sigma \sqsubseteq \mu^{\prime} \sqsubseteq \mu \triangleright\left(g \xi \sqcup \xi^{\sharp}\right)$, we have $\sup (\sigma, \xi) \sqsubseteq \mu \triangleright\left(g \xi \sqcup \xi^{\sharp}\right)$ by Proposition 3.5 and so $\sup (\sigma, \xi) \sqsubseteq \mu^{\prime}$. 
(T2) We will see $\mu^{\prime} g \sqsubseteq \mu^{\prime}$. By the definition of $\mu$ and Galois connection $\tau_{0} \sqsubseteq \mu \triangleright\left(\xi \sqcup \xi^{\sharp}\right)$ holds. Hence we have

$$
\begin{array}{rlll}
\mu^{\prime} g & =\left(\tau_{0} \sqcap\left(\mu \triangleright\left(g \xi \sqcup \xi^{\sharp}\right)\right)\right) g & & \\
& \sqsubseteq \tau_{0} g \sqcap\left(\mu \triangleright\left(g \xi g \sqcup \xi^{\sharp} g\right)\right) & & \{2.3(\mathrm{i})\} \\
& \sqsubseteq \tau_{0} \sqcap\left(\mu \triangleright\left(g \xi \sqcup \xi^{\sharp} g\right)\right) & & \left\{\tau_{0} g \sqsubseteq \tau_{0}, g \sqsubseteq \xi\right\} \\
& \left.=\tau_{0} \sqcap\left(\mu \triangleright\left(\xi \sqcup \xi^{\sharp}\right)\right) \sqcap\left(\mu \triangleright\left(g \xi \sqcup \xi^{\sharp} g\right)\right)\right) & & \left\{\tau_{0} \sqsubseteq \mu \triangleright\left(\xi \sqcup \xi^{\sharp}\right)\right\} \\
& =\tau_{0} \sqcap\left(\mu \triangleright\left(\left(\xi \sqcup \xi^{\sharp}\right) \sqcap\left(g \xi \sqcup \xi^{\sharp} g\right)\right)\right) & & \{2.3(\mathrm{j})\} \\
& \sqsubseteq \tau_{0} \sqcap\left(\mu \triangleright\left(g \xi \sqcup \xi^{\sharp}\right)\right) & & \{3.3\} \\
& =\mu^{\prime} . & &
\end{array}
$$

(b) (T1) Let $\sigma: V \rightarrow X$ be a $\xi$-chain with $\sigma \sqsubseteq \mu=\tau_{0} \triangleright\left(\xi^{\sharp} \sqcup \xi\right)$. Then by Proposition 3.5 (note $\left.g=\operatorname{id}_{X}\right)$ we have $\sup (\sigma, \xi) \sqsubseteq \mu$.

(T2) We will see $\mu g \sqsubseteq \mu$. As $\mu^{\prime}$ is a $(\xi, g)$-tower by (a) and $\tau_{0}$ is the minimum $(\xi, g)$ tower, it holds that $\tau_{0} \sqsubseteq \mu^{\prime} \sqsubseteq \mu \triangleright\left(g \xi \sqcup \xi^{\sharp}\right)$ and so $\mu \sqsubseteq \tau_{0} \triangleright\left(\xi^{\sharp} g^{\sharp} \sqcup \xi\right)$ by the Galois connection. Hence we have

$$
\begin{array}{rlrl}
\mu g & \sqsubseteq\left(\tau_{0} \triangleright\left(\xi^{\sharp} g^{\sharp} \sqcup \xi\right)\right) g & \\
& \sqsubseteq \tau_{0} \triangleright\left(\xi^{\sharp} g^{\sharp} g \sqcup \xi g\right) & & \{2.3(\mathrm{i})\} \\
& \sqsubseteq \tau_{0} \triangleright\left(\xi^{\sharp} \sqcup \xi\right) & & \left\{g^{\sharp} g \sqsubseteq \mathrm{id}_{X}, g \sqsubseteq \xi\right\} \\
& =\mu . & &
\end{array}
$$

(c) Since $\mu$ is a $(\xi, g)$-tower from (b), the inclusion $\tau_{0} \sqsubseteq \mu$ holds by the minimality of $\tau_{0}$, which means that $\tau_{0}$ is a $\xi$-chain.

(d) As the minimum $(\xi, g)$-tower $\tau_{0}$ is a $\xi$-chain by (c) we have $\sup \left(\tau_{0}, \xi\right) g \sqsubseteq \tau_{0} g \sqsubseteq \tau_{0}$ by the conditions (T1) and (T2), and so

$$
\begin{aligned}
\sup \left(\tau_{0}, \xi\right) g & \sqsubseteq \tau_{0} \sqcap \sup \left(\tau_{0}, \xi\right) \xi & & \left\{\sup \left(\tau_{0}, \xi\right) g \sqsubseteq \tau_{0}, g \sqsubseteq \xi\right\} \\
& \sqsubseteq \tau_{0} \sqcap\left(\tau_{0} \triangleright \xi\right) & & \left.\sup \left(\tau_{0}, \xi\right) \sqsubseteq \tau_{0} \triangleright \xi\right\} \\
& =\max \left(\tau_{0}, \xi\right) . & &
\end{aligned}
$$

(e) By the result of (d) we have $\sup \left(\tau_{0}, \xi\right) g \sqsubseteq \max \left(\tau_{0}, \xi\right) \sqsubseteq \sup \left(\tau_{0}, \xi\right)$. Because of the chain completeness of $\xi$, the supremum $\sup \left(\tau_{0}, \xi\right)$ is a function and so the desired equality holds by Proposition 2.3 (f).

\section{Cantor categories}

Roughly speaking, a Cantor category is a Dedekind category possessing rational representations and membership relations. A rational representation (or tabulation) of $\alpha$ is a pair of functions $f: R \rightarrow X$ and $g: R \rightarrow Y$ such that $\alpha=f^{\sharp} g$ and $f f^{\sharp} \sqcap g g^{\sharp}=\mathrm{id}_{R}$. If a universal relation $\nabla_{X Y}$ in a Dedekind category has a rational representation, we call the pair projections and the domain of the projections is denoted by $X \times Y$ analogously. An object $\wp(Y)$ and a relation $\ni_{Y}: \wp(Y) \rightarrow Y$ satisfying

$(\mathrm{M} 1) \quad\left(\ni_{Y} \triangleright \ni_{Y}^{\sharp}\right) \sqcap\left(\ni_{Y} \triangleright \ni_{Y}^{\sharp}\right)^{\sharp} \sqsubseteq \operatorname{id}_{\wp(Y)}$,

$(\mathrm{M} 2) \operatorname{id}_{X} \sqsubseteq\left(\alpha \triangleright \ni_{Y}^{\sharp}\right)\left(\ni_{Y} \triangleright \alpha^{\sharp}\right)$ for all relations $\alpha: X \rightarrow Y$

are called a power object of an object $Y$ and a membership relation for an object $Y$, respectively. 
Definition 4.1. A Dedekind category is called a Cantor category if it satisfies the following two conditions.

(Rat) Every relation $\alpha: X \rightarrow Y$ has a rational representation,

(Pow) Every object $Y$ has a membership relation $\ni_{Y}: \wp(Y) \rightarrow Y$.

The above conditions (Rat) and (Pow) are called the axiom of rationality and the axiom of power objects, respectively. Note that a Cantor category has units without any assumptions.

Example 4.2. The Dedekind category Rel is a Cantor category since

- each relation $\alpha \in \operatorname{Rel}(X, Y)$ has a rational representation $i p: \alpha \rightarrow X$ and $i q: \alpha \rightarrow$ $Y$, where $i: \alpha \rightarrow X \times Y$ is the inclusion and $p: X \times Y \rightarrow X$ and $q: X \times Y \rightarrow Y$ are the projections, and

- the power set $\wp(Y)$ and the membership relation $\ni_{Y}=\{(S, y) \in \wp(Y) \times Y \mid y \in S\}$ satisfy (M1) and (M2) in Rel.

For all relations $\alpha: X \rightarrow Y$ in a Cantor category define a relation $\alpha^{@}: X \rightarrow \wp(Y)$ by

$$
\alpha^{@}=\left(\alpha \triangleright \ni_{Y}^{\sharp}\right) \sqcap\left(\ni_{Y} \triangleright \alpha^{\sharp}\right)^{\sharp} .
$$

Then $\alpha^{@}$ is a function, that is, it is univalent by (M1) and total by (M2). Moreover $\alpha^{@}$ is a unique function such that $\alpha^{@} \ni_{Y}=\alpha$, and this leads the power adjunction in a sense that a relation $\alpha: X \rightarrow Y$ bijectively corresponds to a function $f: X \rightarrow \wp(Y): \alpha=f \ni_{Y}$ if and only if $f=\alpha^{@}$. This correspondence is denoted by the following notation.

$$
\frac{\alpha: X \rightarrow Y}{f: X \rightarrow \wp(Y)} \quad \frac{\alpha=f \ni_{Y}}{f=\alpha^{@}} \quad \text { (power adjunction) }
$$

For a relation $\alpha: X \rightarrow Y$ define a function $\wp(\alpha): \wp(X) \rightarrow \wp(Y)$ by $\wp(\alpha)=\left(\ni_{X} \alpha\right)^{@}$. By the power adjunction $\wp(\alpha)$ is a unique function such that the following diagram commutes.

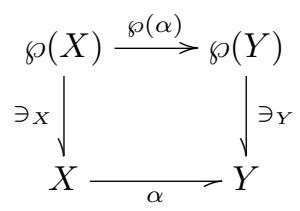

For an object $X, \wp\left(\operatorname{id}_{X}\right) \ni_{X}=\ni_{X} \operatorname{id}_{X}=\operatorname{id}_{\wp(X)} \ni_{X}$ holds. Thus $\wp$ preserves identities by the uniqueness. Also, for relations $\alpha: X \neg Y$ and $\beta: Y \rightarrow X, \wp(\alpha \beta) \ni_{Z}=\ni_{X} \alpha \beta=$ $\wp(\alpha) \ni_{Y} \beta=\wp(\alpha) \wp(\beta) \ni_{Z}$ holds. Therefore, again by the uniqueness, $\wp$ preserves composition. The power order $\Xi_{X}: \wp(X) \rightarrow \wp(X)$ is defined by

$$
\Xi_{X}=\ni_{X} \triangleright \ni_{X}^{\sharp}
$$

Obviously the power order $\Xi_{X}$ is in fact an order, since it is reflexive and transitive by the definition, and antisymmetric by (M1).

Example 4.3. Consider the Dedekind category Rel. Then, 
- a relation $\alpha: X \rightarrow Y$ corresponds to the relation $\alpha^{@}: X \rightarrow \wp(Y)$ defined by $(x, S) \in \alpha^{@}$ if and only if $S=\{y \in Y \mid(x, y) \in \alpha\}$ by the power adjunction,

- $\wp(\alpha): \wp(X) \rightarrow \wp(Y)$ maps $A$ to $\{b \in Y \mid \exists a \in A$. $(a, b) \in \alpha\}$, and

- $\Xi_{X}=\left\{\left(S, S^{\prime}\right) \in \wp(X) \times \wp(X) \mid S \subseteq S^{\prime}\right\}$ is the power order.

The power order satisfies the following properties in any Cantor categories.

Proposition 4.4. In Cantor categories the following statements hold.

(a) $\ni_{\wp(X)} \triangleright \Xi_{X}=\wp\left(\ni_{X}\right) \Xi_{X}$.

(b) $\sup \left(\ni_{\wp(X)}, \Xi_{X}\right)=\wp\left(\ni_{X}\right)$.

(c) $\sup \left(\rho, \Xi_{X}\right)=\left(\rho \ni_{X}\right)^{\circledR}$ for all relations $\rho: V \neg \wp(X)$.

(d) $\Xi_{X}$ is complete on all objects $V$.

Proof. (a) $\ni_{\wp(X)} \triangleright \Xi_{X}=\wp\left(\ni_{X}\right) \Xi_{X}$ follows from

$$
\begin{aligned}
\ni_{\wp(X)} \triangleright \Xi_{X} & =\ni_{\wp(X)} \triangleright\left(\ni_{X} \triangleright \ni_{X}^{\sharp}\right) & \\
& =\ni_{\wp(X)} \ni_{X} \triangleright \ni_{X}^{\sharp} & \{2.3(\mathrm{i})\} \\
& =\wp\left(\ni_{X}\right) \ni_{X} \triangleright \ni_{X}^{\sharp} & \\
& =\wp\left(\ni_{X}\right)\left(\ni_{X} \triangleright \ni_{X}^{\sharp}\right) & \{2.3(\mathrm{n})\} \\
& =\wp\left(\ni_{X}\right) \Xi_{X} . &
\end{aligned}
$$

(b) $\sup \left(\ni_{\wp(X)}, \Xi_{X}\right)=\wp\left(\ni_{X}\right)$ follows from

$$
\begin{aligned}
\sup \left(\ni_{\wp(X)}, \Xi_{X}\right) & =\wp\left(\ni_{X}\right) \Xi_{X} \sqcap\left(\wp\left(\ni_{X}\right) \Xi_{X} \triangleright \Xi_{X}^{\sharp}\right) & & \{(\mathrm{a})\} \\
& =\wp\left(\ni_{X}\right) \Xi_{X} \sqcap \wp\left(\ni_{X}\right)\left(\Xi_{X} \triangleright \Xi_{X}^{\sharp}\right) & & \{2.3(\mathrm{n})\} \\
& =\wp\left(\ni_{X}\right) \Xi_{X} \sqcap \wp\left(\ni_{X}\right) \Xi_{X}^{\sharp} & & \left\{\Xi_{X}: \text { order }\right\} \\
& =\wp\left(\ni_{X}\right)\left(\Xi_{X} \sqcap \Xi_{X}^{\sharp}\right) & & \{2.3(\mathrm{~d})\} \\
& =\wp\left(\ni_{X}\right) . & & \left\{\Xi_{X}: \text { order }\right\}
\end{aligned}
$$

(c) $\sup \left(\rho, \Xi_{X}\right)=\left(\rho \ni_{X}\right)^{@}$ follows from

$$
\begin{aligned}
\sup \left(\rho, \Xi_{X}\right) & =\sup \left(\rho^{@} \ni_{\wp(X)}, \Xi_{X}\right) & & \left\{\rho=\rho^{@} \ni_{\wp(X)}\right\} \\
& =\rho^{@} \sup \left(\ni_{\wp(X)}, \Xi_{X}\right) & & \{2.7(\mathrm{~d})\} \\
& =\rho^{@} \wp\left(\ni_{X}\right) & & \{(\mathrm{b})\} \\
& =\left(\rho \ni_{X}\right)^{@} . & &
\end{aligned}
$$

(d) It is a corollary of (c).

For relations $\alpha: X \neg Y, \beta: Y \rightarrow Z$ and $\gamma: Z \rightarrow W$ in a Dedekind category two sided residual composite $\alpha \triangleright \beta \triangleleft \gamma: X \neg W$ is defined by $\alpha \triangleright \beta \triangleleft \gamma=\alpha \triangleright\left(\gamma^{\sharp} \triangleright \beta^{\sharp}\right)^{\sharp}$. The two sided residual composite is characterised by an equivalence

$$
\delta \sqsubseteq \alpha \triangleright \beta \triangleleft \gamma \leftrightarrow \alpha^{\sharp} \delta \gamma^{\sharp} \sqsubseteq \beta
$$

for all relations $\delta: X \neg W$. Remark that the identity $\alpha \triangleright \beta \triangleleft \gamma=\left(\alpha \beta^{-} \gamma\right)^{-}$holds in Schröder categories. 
The next lemma gives a construction of chain complete orders from the power order. The construction extends a well-known fact that the set of all chains in an ordered set forms a chain complete ordered set. Note that the relation $i \Xi_{X} i^{\sharp}: C \rightarrow C$ is an order on $C$ for the power order $\Xi_{X}$ and an injection $i: C \rightarrow \wp(X)$.

Lemma 4.5. Let $\delta: X \rightarrow X$ be a relation. If $i: C \rightarrow \wp(X)$ is an injection such that $i^{\sharp} i=\operatorname{id}_{\wp(X)} \sqcap\left(\ni_{X} \triangleright \delta \triangleleft \ni_{X}^{\sharp}\right)$, then the induced order $\Xi_{i}=i \Xi_{X} i^{\sharp}$ is chain complete on all objects $V$.

Proof. Let $\sigma: V \rightarrow C$ be a $\Xi_{i}$-chain.

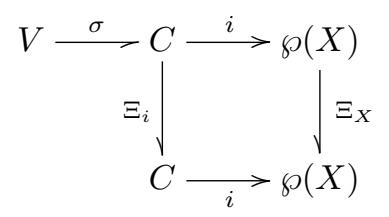

By $4.4(\mathrm{c}) s=\sup \left(\sigma i, \Xi_{X}\right)$ is a function such that $s \ni_{X}=\sigma i \ni_{X}$. The totality of $s i^{\sharp}$ follows (1) and (2) below.

(1) $s^{\sharp} s \sqsubseteq i i^{\sharp} i$ :

$$
\begin{aligned}
\ni_{X}^{\sharp} s^{\sharp} s \ni_{X} & =\ni_{X}^{\sharp} i^{\sharp} \sigma^{\sharp} \sigma i \ni_{X} & & \left\{s \ni_{X}=\sigma i \ni_{X}\right\} \\
& \sqsubseteq \ni_{X}^{\sharp} i^{\sharp} \Xi_{i} \Xi_{i}^{\sharp} i \ni_{X} & & \left\{\sigma^{\sharp} \sigma \Xi_{i} \sqcup \Xi_{i}^{\sharp} \Xi_{i} \Xi_{i}^{\sharp}\right\} \\
& \sqsubseteq \ni_{X}^{\sharp} i^{\sharp} i \ni_{X} & & \left\{\Xi_{i}^{\sharp} i \ni_{X} \sqsubseteq i \ni_{X}\right\} \\
& \sqsubseteq \delta . & & \left\{i^{\sharp} i \sqsubseteq \ni_{X} \triangleright \delta \triangleleft \ni_{X}^{\sharp}\right\}
\end{aligned}
$$

(2) $s i \sharp$ is total:

$$
\begin{aligned}
\operatorname{id}_{V} & \sqsubseteq s s^{\sharp} s s^{\sharp} & \{s: \text { function }\} \\
& =s i^{\sharp} i s^{\sharp} . & \left\{(1) s^{\sharp} s \sqsubseteq i^{\sharp} i\right\}
\end{aligned}
$$

Hence $\sup \left(\sigma, \Xi_{i}\right)$ is total, since $s i^{\sharp} \sqsubseteq \sup \left(\sigma, \Xi_{i}\right)$ holds by 2.7 (f).

The following proposition gives a relational construction of the set of all chains in an ordered set.

Proposition 4.6. Let $\xi: X \rightarrow X$ be a reflexive relation and $i: C \rightarrow \wp(X)$ an injection such that $i^{\sharp} i=\operatorname{id}_{\wp(X)} \sqcap\left(\ni_{X} \triangleright\left(\xi \sqcup \xi^{\sharp}\right) \triangleleft \ni_{X}^{\sharp}\right)$. Then

(a) For all univalent relations $s: V \rightarrow C$ the composite si $\ni_{X}$ is a $\xi$-chain.

(b) For all univalent relations $f: C \rightarrow X$ satisfying $f \sqsubseteq i \ni_{X} \triangleright \xi$ there exists a function $g: C \rightarrow C$ such that $g i \ni_{X}=i \ni_{X} \sqcup f$.

Proof. (a) Let $s: V \rightarrow C$ be a univalent relation. Then

$$
\begin{aligned}
\left(s i \ni_{X}\right)^{\sharp}\left(s i \ni_{X}\right) & =\ni_{X}^{\sharp} i^{\sharp} s^{\sharp} s i \ni_{X} & & \\
& \sqsubseteq \ni_{X}^{\sharp} i^{\sharp} i \ni_{X} & & \left\{s^{\sharp} s \sqsubseteq \operatorname{id}_{C}\right\} \\
& \sqsubseteq \xi \sqcup \xi^{\sharp}, & & \left\{i^{\sharp} i \sqsubseteq \ni_{X} \triangleright\left(\xi \sqcup \xi^{\sharp}\right) \triangleleft \ni_{X}^{\sharp}\right\}
\end{aligned}
$$


which shows that $s i \ni_{X}$ is a $\xi$-chain.

(b) Set $h=\left(i \ni_{X} \sqcup f\right)^{@}: C \rightarrow \wp(X)$. Then $h^{\sharp} h \sqsubseteq i^{\sharp} i$ follows from

$$
\begin{aligned}
\ni_{X}^{\sharp} h h^{\sharp} h \ni_{X} & =\left(i \ni_{X} \sqcup f\right)^{\sharp}\left(i \ni_{X} \sqcup f\right) & & \left\{h \ni_{X}=i \ni_{X} \sqcup f\right\} \\
& =\left(i \ni_{X}\right)^{\sharp} i \ni_{X} \sqcup\left(i \ni_{X}\right)^{\sharp} f \sqcup f^{\sharp} i \ni_{X} \sqcup f^{\sharp} f & & \\
& \sqsubseteq \xi \sqcup \xi^{\sharp} \sqcup\left(i \ni_{X}\right)^{\sharp} f \sqcup f^{\sharp} i \ni_{X} \sqcup f^{\sharp} f & & \left\{\left(i \ni_{X}\right)^{\sharp} i \ni_{X} \sqsubseteq \xi \sqcup \xi^{\sharp}\right\} \\
& \sqsubseteq \xi \sqcup \xi^{\sharp} \sqcup \operatorname{id}_{X} & & \left\{\sqsubseteq i \ni_{X} \triangleright \xi\right\} \\
& =\xi \sqcup \xi^{\sharp} . & & \left\{\operatorname{id}_{X} \sqsubseteq \xi\right\}
\end{aligned}
$$

Hence by the injection adjunction there is a unique function $g$ such that $g i=h$. This completes the proof.

In the rest of this paper, we abbreviate Cantor categories satisfying the axiom of complements (Ba) (or equivalently Schröder categories satisfying the axiom of rationality (Rat) and the axiom of power objects (Pow)) just as Cantor categories.

\section{The equivalence}

With the fixpoint theorem stated in Section 3 we are ready to prove (ZL) from $\left(\mathrm{AC}_{*}\right)$ in Cantor categories.

The next lemma shows a fundamental idea how to construct $\xi$-maximal function for an order $\xi$.

Lemma 5.1. Let $\xi: X \rightarrow X$ be an order and $\sigma: V \rightarrow X$ a relation. If $\sigma \triangleright \xi$ is total and $\sigma \triangleright \xi \sqsubseteq \sigma$ holds, then $\sigma \triangleright \xi$ is a $\xi$-maximal function.

Proof. Assume that $\sigma \triangleright \xi$ is total and $\sigma \triangleright \xi \sqsubseteq \sigma$. Then the equation $\max (\sigma, \xi)=$ $\sigma \sqcap(\sigma \triangleright \xi)=\sigma \triangleright \xi$ holds. Since $\xi$ is antisymmetric, $\max (\sigma, \xi)$ is univalent by $2.7(\mathrm{e})$. Hence $\sigma \triangleright \xi$ is a function. Moreover, it follows from 2.3 (i) and the transitivity of $\xi$ that $(\sigma \triangleright \xi) \xi \sqsubseteq \sigma \triangleright \xi \xi \sqsubseteq \sigma \triangleright \xi$, which proves that $\sigma \triangleright \xi$ is $\xi$-maximal.

Theorem 5.2. In Cantor categories $\left(\mathrm{AC}_{*}\right)$ implies (ZL).

Proof. Assume that an order $\xi: X \neg X$ is inductive on $V$. By the axiom of rationality (Rat) and $\operatorname{id}_{\wp(X)} \sqcap\left(\ni_{X} \triangleright\left(\xi \sqcup \xi^{\sharp}\right) \triangleleft \ni_{X}^{\sharp}\right) \sqsubseteq \operatorname{id}_{\wp(X)}$ there is an injection $i: C \rightarrow \wp(X)$ such that

$$
i^{\sharp} i=\operatorname{id}_{\wp(X)} \sqcap\left(\ni_{X} \triangleright\left(\xi \sqcup \xi^{\sharp}\right) \triangleleft \ni_{X}^{\sharp}\right) .
$$

The induced order $\Xi_{i}=i \Xi_{X} i^{\sharp}: C \rightarrow C$ is chain complete by Lemma 4.5. Define a relation $\alpha: C \rightarrow X$ by

$$
\alpha=\left(i \ni_{X} \triangleright \xi\right) \sqcap\left(i \ni_{X}\right)^{-} .
$$

Then by $\left(\mathrm{AC}_{*}\right)$ there is a univalent relation $\gamma: C \rightarrow X$ such that $\gamma \sqsubseteq \alpha$ and $\lfloor\gamma\rfloor=\lfloor\alpha\rfloor$.

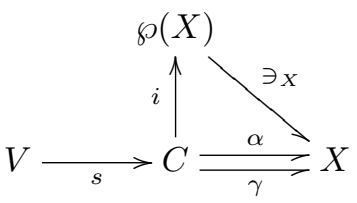


As $\gamma \sqsubseteq i \ni_{X} \triangleright \xi$, by Proposition 4.6 (b) there exists a function $g: C \rightarrow C$ such that $g i \ni_{X}=i \ni_{X} \sqcup \gamma$. Moreover, $g$ is a $\Xi_{i}$-section by Proposition 3.6. Hence by the fixpoint theorem $3.9(\mathrm{e})$ there exists a function $s: V \rightarrow C$ such that $s g=s$. The identity $s \alpha=0_{V X}$ follows from

$$
\begin{array}{rlll}
s \alpha & =s\lfloor\alpha\rfloor \alpha & & \{\alpha=\lfloor\alpha\rfloor \alpha\} \\
& \sqsubseteq s \gamma \gamma^{\sharp} \alpha & & \left\{\lfloor\rfloor=\lfloor\gamma\rfloor \sqsubseteq \gamma \gamma^{\sharp}\right\} \\
& \sqsubseteq\left(s g i \ni_{X} \sqcap s\left(i \ni_{X}\right)^{-}\right) \gamma^{\sharp} \alpha & & \left\{\gamma \sqsubseteq g i \ni_{X}, \gamma \sqsubseteq \alpha \sqsubseteq\left(i \ni_{X}\right)^{-}\right\} \\
& =\left(s i \ni_{X} \sqcap\left(s i \ni_{X}\right)^{-}\right) \gamma^{\sharp} \alpha & & \{s g=s, s: \text { function }\} \\
& =0_{V X} . & &
\end{array}
$$

On the other hand we have

$$
\begin{array}{rlrl}
s \alpha & =s\left(\left(i \ni_{X} \triangleright \xi\right) \sqcap\left(i \ni_{X}\right)^{-}\right) & & \{\text {Def. of } \alpha\} \\
& =\left(s i \ni_{X} \triangleright \xi\right) \sqcap\left(s i \ni_{X}\right)^{-}, & \{s: \text { function }\}
\end{array}
$$

which shows $s i \ni_{X} \triangleright \xi \sqsubseteq s i \ni_{X}$. Also $s i \ni_{X}$ is a $\xi$-chain by Proposition 4.6 (a) and $\operatorname{si} \ni_{X} \triangleright \xi$ is total since $\xi$ is inductive on $V$. Hence by the virtue of Lemma 5.1 si $\ni_{X} \triangleright \xi$ is a $\xi$-maximal function. This completes the proof.

Next we will show that Zorn's lemma conversely implies the axiom of choice in Cantor categories. The relational proof exposes a construction of an object representing the set of all graphs of univalent relations, and the restricted axiom of choice (AC- $I$ ) guaranteeing to pick up a point of a nonempty set.

In the rest of this section we assume that $\alpha: X \rightarrow Y$ is a relation in a Cantor category and a pair of functions $p: R \rightarrow X$ and $q: R \rightarrow Y$ is a rational representation of $\alpha$, which exists by the axiom of rationality (Rat): $\alpha=p^{\sharp} q$ and $p p^{\sharp} \sqcap q q^{\sharp}=\operatorname{id}_{R}$. Also for relations $\gamma: X \rightarrow Y$ such that $\gamma \sqsubseteq \alpha$ and $\nu: I \rightarrow R$ relations $\phi(\gamma): I \rightarrow R$ and $\psi(\nu): X \neg Y$ are respectively defined by $\phi(\gamma)=\nabla_{I X}\left(p^{\sharp} \sqcap \gamma q^{\sharp}\right)$ and $\psi(\nu)=\left(\nabla_{X I} \nu \sqcap p^{\sharp}\right) q$.

Proposition 5.3. The following statements hold.

(a) A relation $\gamma: X \rightarrow Y$ such that $\gamma \sqsubseteq \alpha$ bijectively corresponds to a relation $\nu: I \rightarrow$ $R: \gamma=\psi(\nu)$ if and only if $\nu=\phi(\gamma)$.

$$
\frac{\gamma: X \neg Y \quad\{\gamma \sqsubseteq \alpha\}}{\nu: I \rightarrow R} \quad \frac{\gamma=\psi(\nu)}{\nu=\phi(\gamma)}
$$

(b) $\phi$ and $\psi$ are isomorphisms of Boolean algebras, that is, $\phi\left(0_{X Y}\right)=0_{I R}, \phi(\alpha)=$ $\nabla_{I R}, \phi\left(\gamma \sqcup \gamma^{\prime}\right)=\phi(\gamma) \sqcup \phi\left(\gamma^{\prime}\right)$ and $\phi\left(\gamma \sqcap \gamma^{\prime}\right)=\phi(\gamma) \sqcap \phi\left(\gamma^{\prime}\right)$.

(c) A univalent relation $\gamma: X \rightarrow Y$ such that $\gamma \sqsubseteq \alpha$ bijectively corresponds to a relation $\nu: I \rightarrow R$ such that $\nu^{\sharp} \nu \sqsubseteq p p^{\sharp} \Rightarrow \operatorname{id}_{R}: \gamma=\psi(\nu)$ if and only if $\nu=\phi(\gamma)$.

$$
\frac{\gamma: X \rightarrow Y \quad\left\{\gamma \sqsubseteq \alpha, \gamma^{\sharp} \gamma \sqsubseteq \operatorname{id}_{Y}\right\}}{\nu: I \rightarrow R \quad\left\{\nu^{\sharp} \nu \sqsubseteq p p^{\sharp} \Rightarrow \operatorname{id}_{R}\right\}} \quad \frac{\gamma=\psi(\nu)}{\nu=\phi(\gamma)}
$$

Proof. Let $\gamma: X \neg Y, \delta: X \neg R$ and $\nu: I \neg R$ be relations and let $\gamma \sqsubseteq \alpha$ and $\delta \sqsubseteq p^{\sharp}$. Defining $\phi_{0}(\gamma)=p^{\sharp} \sqcap \gamma q^{\sharp}$ and $\phi_{1}(\delta)=\nabla_{I X} \delta$, we have $\phi(\gamma)=\phi_{1}\left(\phi_{0}(\gamma)\right)$. 
Similarly, defining $\psi_{0}(\delta)=\delta q$ and $\psi_{1}(\nu)=\nabla_{X I} \nu \sqcap p^{\sharp}$ we have $\psi_{0}(\delta)=\delta q \sqsubseteq p^{\sharp} q=\alpha$ and $\psi(\nu)=\psi_{0}\left(\psi_{1}(\nu)\right)$. Using these decompositions, we show (a), (b) and (c).

(a) It is sufficient to show that the following bijective correspondences

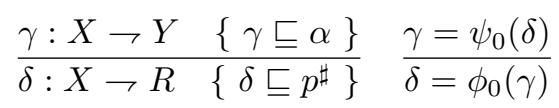

and

$$
\frac{\delta: X \neg R \quad\left\{\delta \sqsubseteq p^{\sharp}\right\}}{\nu: I \neg R} \quad \frac{\delta=\psi_{1}(\nu)}{\nu=\phi_{1}(\delta)}
$$

hold. $\psi_{0}\left(\phi_{0}(\gamma)\right)=\left(\gamma q^{\sharp} \sqcap p^{\sharp}\right) q=\gamma$ follows from

$$
\begin{aligned}
\gamma & =\gamma \sqcap p^{\sharp} q & & \left\{\gamma \sqsubseteq \alpha=p^{\sharp} q\right\} \\
& \sqsubseteq\left(\gamma q^{\sharp} \sqcap p^{\sharp}\right) q & & \{\mathrm{DF}\} \\
& \sqsubseteq \gamma q^{\sharp} q & & \\
& \sqsubseteq \gamma . & & \left\{q^{\sharp} q \sqsubseteq \operatorname{id}_{Y}\right\}
\end{aligned}
$$

On the other hand $\phi_{0}\left(\psi_{0}(\delta)\right)=\delta q q^{\sharp} \sqcap p^{\sharp}=\delta$ follows from

$$
\begin{aligned}
& \delta=\delta \sqcap p^{\sharp} \quad\left\{\delta \sqsubseteq p^{\sharp}\right\} \\
& \sqsubseteq \delta q q^{\sharp} \sqcap p^{\sharp} \quad\left\{\operatorname{id}_{Y} \sqsubseteq q q^{\sharp}\right\} \\
& \sqsubseteq \delta\left(q q^{\sharp} \sqcap \delta^{\sharp} p^{\sharp}\right) \quad\{\mathrm{DF}\} \\
& \sqsubseteq \delta\left(q q^{\sharp} \sqcap p p^{\sharp}\right) \quad\left\{\delta \sqsubseteq p^{\sharp}\right\} \\
& =\delta . \quad\left\{p p^{\sharp} \sqcap q q^{\sharp}=\mathrm{id}_{R}\right\}
\end{aligned}
$$

So, the first bijective correspondence holds. For the second $\psi_{1}\left(\phi_{1}(\delta)\right)=\nabla_{X I} \nabla_{I X} \delta \sqcap p^{\sharp}=$ $\delta$ follows from

$$
\begin{array}{rlrl}
\delta & =\delta \sqcap p^{\sharp} & & \left\{\delta \sqsubseteq p^{\sharp}\right\} \\
\sqsubseteq \nabla_{X X} \delta \sqcap p^{\sharp} & \left\{\mathrm{id}_{X} \sqsubseteq \nabla_{X X}=\nabla_{X I} \nabla_{I X}\right\} \\
\sqsubseteq\left(\nabla_{X X} \sqcap p^{\sharp} \delta^{\sharp}\right) \delta & \{\mathrm{DF}\} \\
\sqsubseteq p^{\sharp} p \delta & \left\{\delta \sqsubseteq p^{\sharp}\right\} \\
\sqsubseteq \delta & \{p: \text { function }\}
\end{array}
$$

and $\phi_{1}\left(\psi_{1}(\nu)\right)=\nabla_{I X}\left(\nabla_{X I} \nu \sqcap p^{\sharp}\right)=\nu$ follows from

$$
\begin{aligned}
\nu & =\nu \sqcap \nabla_{I X} p^{\sharp} & & \left\{\nabla_{I R}=\nabla_{I X} p^{\sharp}\right\} \\
& \sqsubseteq \nabla_{I X}\left(\nabla_{X I} \nu \sqcap p^{\sharp}\right) & & \{\mathrm{DF}\} \\
& \sqsubseteq \nabla_{I X} \nabla_{X I} \nu & & \\
& \sqsubseteq \nabla_{I I} \nu & & \left\{\nabla_{I X} \nabla_{X I} \sqsubseteq \nabla_{I I}\right\} \\
& =\nu . & &
\end{aligned}
$$

(b) It trivial that $\phi_{0}\left(0_{X Y}\right)=0_{X R}, \phi_{0}\left(\gamma \sqcup \gamma^{\prime}\right)=\phi_{0}(\gamma) \sqcup \phi_{0}\left(\gamma^{\prime}\right), \phi_{0}\left(\gamma \sqcap \gamma^{\prime}\right)=\phi_{0}(\gamma) \sqcap \phi_{0}\left(\gamma^{\prime}\right)$, $\phi_{1}\left(0_{X R}\right)=0_{I R}$, and $\phi_{1}\left(\delta \sqcup \delta^{\prime}\right)=\phi_{1}(\delta) \sqcup \phi_{1}\left(\gamma^{\prime}\right)$. Also $\phi_{1}\left(p^{\sharp}\right)=\nabla_{I R}$ follows from

$$
\begin{aligned}
\nabla_{I R} & \sqsubseteq \nabla_{I R} p p^{\sharp} \quad\left\{\operatorname{id}_{R} \sqsubseteq p p^{\sharp}\right\} \\
& \sqsubseteq \nabla_{I X} p^{\sharp} \\
& =\phi_{1}\left(p^{\sharp}\right),
\end{aligned}
$$


and $\phi_{1}\left(\delta \sqcap \delta^{\prime}\right)=\phi_{1}(\delta) \sqcap \phi_{1}\left(\gamma^{\prime}\right)$ follows from

$$
\begin{array}{rlrl}
\nabla_{I X} \delta \sqcap \nabla_{I X} \delta^{\prime} & \sqsubseteq \nabla_{I X}\left(\delta \sqcap \nabla_{X I} \nabla_{I X} \delta^{\prime}\right) & & \{\mathrm{DF}\} \\
& =\nabla_{I X}\left(\delta \sqcap \nabla_{X X} \delta^{\prime}\right) & & \left\{\nabla_{X I} \nabla_{I X}=\nabla_{X X}\right\} \\
& =\nabla_{I X}\left(\delta \sqcap p^{\sharp} \sqcap \nabla_{X X} \delta^{\prime}\right) & & \left\{\delta \sqsubseteq p^{\sharp}\right\} \\
& \sqsubseteq \nabla_{I X}\left(\delta \sqcap\left(p^{\sharp} \delta^{\prime} \sqcap \sqcap \nabla_{X X}\right) \delta^{\prime}\right) & \{\mathrm{DF}\} \\
& \sqsubseteq \nabla_{I X}\left(\delta \sqcap p^{\sharp} p \delta^{\prime}\right) & & \left\{\delta^{\prime} \sqsubseteq p^{\sharp}\right\} \\
& \sqsubseteq \nabla_{I X}\left(\delta \sqcap \delta^{\prime}\right) . & & \left\{p^{\sharp} \sqsubseteq \operatorname{id}_{X}\right\}
\end{array}
$$

(c) Let $\phi(\gamma)=\nu$. Then it is sufficient to show the following equivalence.

$$
\gamma \sqsubseteq \alpha \wedge \gamma^{\sharp} \gamma \sqsubseteq \operatorname{id}_{Y} \leftrightarrow \nu^{\sharp} \nu \sqsubseteq p p^{\sharp} \Rightarrow \operatorname{id}_{R}
$$

To see it we need to prove $\gamma^{\sharp} \gamma=q^{\sharp}\left(\nu^{\sharp} \nu \sqcap p p^{\sharp}\right) q$. This equation follows from

$$
\gamma^{\sharp} \gamma=\psi_{0}\left(\phi_{0}(\gamma)\right)^{\sharp} \psi_{0}\left(\phi_{0}(\gamma)\right)=\left(\phi_{0}(\gamma) q\right)^{\sharp} \phi_{0}(\gamma) q=q^{\sharp} \phi_{0}(\gamma)^{\sharp} \phi_{0}(\gamma) q
$$

and

$$
\begin{array}{lll} 
& \phi_{0}(\gamma)^{\sharp} \phi_{0}(\gamma) & \\
=\phi_{0}(\gamma)^{\sharp} \phi_{0}(\gamma) \sqcap p p^{\sharp} & \left\{\phi_{0}(\gamma) \sqsubseteq p^{\sharp}\right\} \\
\sqsubseteq \phi_{0}(\gamma)^{\sharp} \nabla_{X I} \nabla_{I X} \phi_{0}(\gamma) \sqcap p p^{\sharp} & \left\{\nabla_{X X}=\nabla_{X I} \nabla_{I X}\right\} \\
=\psi_{0}\left(\phi_{0}(\gamma)\right)^{\sharp} \psi_{0}\left(\phi_{0}(\gamma)\right) \sqcap p p^{\sharp} & \left\{\text { Def. of } \psi_{0}\right\} \\
=\phi_{0}(\gamma)^{\sharp} \nabla_{X X} \phi_{0}(\gamma) \sqcap p p^{\sharp} & \left\{\nabla_{X X}=\nabla_{X I} \nabla_{I X}\right\} \\
\sqsubseteq \phi_{0}(\gamma)^{\sharp}\left(\nabla_{X X} \sqcap \phi_{0}(\gamma) p p^{\sharp} \phi_{0}(\gamma)^{\sharp}\right) \phi_{0}(\gamma) & \{\text { DF }\} \\
\sqsubseteq \phi_{0}(\gamma)^{\sharp} p^{\sharp} p p^{\sharp} p \phi_{0}(\gamma) & \left\{\phi_{0}(\gamma) \sqsubseteq p^{\sharp}\right\} \\
\sqsubseteq \phi_{0}(\gamma)^{\sharp} \phi_{0}(\gamma) . & \left\{p^{\sharp} \sqsubseteq \operatorname{id}_{X}\right\}
\end{array}
$$

If $\gamma \sqsubseteq \alpha$, there exists a unique relation $\nu: I \rightarrow R$ such that $\gamma=\psi(\nu)$ by the last proposition and $\lfloor\gamma\rfloor=\nabla_{X I} \nu p \sqcap \mathrm{id}_{X}$ holds by

$$
\begin{aligned}
\lfloor\gamma\rfloor & =\lfloor\psi(\nu)\rfloor \\
& =\left\lfloor\left(\nabla_{X I} \nu \sqcap p^{\sharp}\right) q\right\rfloor \quad \\
& =\left\lfloor\nabla_{X I} \nu \sqcap p^{\sharp}\right\rfloor \quad\{q: \text { function }\} \\
& =\nabla_{X I} \nu p \sqcap \operatorname{id}_{X} .
\end{aligned}
$$

Next, applying Lemma 4.5, we construct an object in a Cantor category, which generalises the set of univalent relations included by a given relation (cf. Corollary 5.5).

In what follows let $j: G \rightarrow \wp(R)$ be an injection such that

$$
j^{\sharp} j=\operatorname{id}_{\wp(R)} \sqcap\left(\ni_{R} \triangleright\left(p p^{\sharp} \Rightarrow \operatorname{id}_{R}\right) \triangleleft \ni_{R}^{\sharp}\right) .
$$

Recall that the pair of the injection $j$ and itself is a rational representation of the relation $\operatorname{id}_{\wp(R)} \sqcap\left(\ni_{R} \triangleright\left(p p^{\sharp} \Rightarrow \operatorname{id}_{R}\right) \triangleleft \ni_{R}^{\sharp}\right)$.

Proposition 5.4. In Cantor categories the following statements hold.

(a) The induced order $\Xi_{j}=j \Xi_{R} j^{\sharp}: G \neg G$ is inductive on all objects $V$, 
(b) A relation $\nu: I \rightarrow R$ such that $\nu^{\sharp} \nu \sqsubseteq p p^{\sharp} \Rightarrow \operatorname{id}_{R}$ bijectively corresponds to a function $x: I \rightarrow G: \nu=x j \ni_{R}$ if and only if $x=\nu^{@} j^{\sharp}$.

$$
\frac{\nu: I \rightarrow R \quad\left\{\nu^{\sharp} \nu \sqsubseteq p p^{\sharp} \Rightarrow \operatorname{id}_{R}\right\}}{x: I \rightarrow G} \quad \frac{\nu=x j \ni_{R}}{x=\nu^{@} j^{\sharp}}
$$

Proof. (a) $\Xi_{j}$ is chain complete by Lemma 4.5 and so inductive by Proposition 2.7 (c).

(b) The desired correspondence is given by the composition of bijective correspondences

$$
\frac{\nu: I \rightarrow R}{t: I \rightarrow \wp(R)} \quad \frac{\nu=t \ni_{R}}{t=\nu^{@}}
$$

(power adjunction)

and

$$
\frac{t: I \rightarrow \wp(R) \quad\left\{t^{\sharp} t \sqsubseteq j^{\sharp} j\right\}}{x: I \rightarrow G} \quad \frac{t=x j}{x=t j^{\sharp}} . \quad \text { (injection adjunction) }
$$

Also, if $\nu=t \ni_{R}$, we have the equivalence $\nu^{\sharp} \nu \sqsubseteq p p^{\sharp} \Rightarrow \operatorname{id}_{R} \leftrightarrow t^{\sharp} t \sqsubseteq j^{\sharp} j$ by

$$
\begin{aligned}
& \nu^{\sharp} \nu \sqsubseteq p p^{\sharp} \Rightarrow \operatorname{id}_{R} \leftrightarrow \ni_{R}^{\sharp} t^{\sharp} t \ni_{R} \sqsubseteq p p^{\sharp} \Rightarrow \operatorname{id}_{R} \quad\left\{\nu=t \ni_{R}\right\} \\
& \leftrightarrow \quad t^{\sharp} t \sqsubseteq \ni_{R} \triangleright\left(p p^{\sharp} \Rightarrow \mathrm{id}_{R}\right) \triangleleft \ni_{R}^{\sharp} \\
& \leftrightarrow \quad t^{\sharp} t \sqsubseteq j^{\sharp} j . \quad\left\{t^{\sharp} t \sqsubseteq \operatorname{id}_{\wp(R)}\right\}
\end{aligned}
$$

This completes the proof.

COROLlary 5.5. A univalent relation $\gamma: X \rightarrow Y$ such that $\gamma \sqsubseteq \alpha$ bijectively corresponds to a function $x: I \rightarrow G: \gamma=\psi\left(x j \ni_{R}\right)$ if and only if $x=(\phi(\gamma))^{@}{ }^{\sharp}$.

$$
\frac{\gamma: X \rightarrow Y \quad\left\{\gamma \sqsubseteq \alpha, \gamma^{\sharp} \gamma \sqsubseteq \mathrm{id}_{Y}\right\}}{x: I \rightarrow G} \quad \frac{\gamma=\psi\left(x j \ni_{R}\right)}{x=(\phi(\gamma))^{@} j^{\sharp}}
$$

ProOF. It is a just a combination of Proposition 5.3 (c) and 5.4 (b).

Now we are ready to deduce $(\mathrm{ZL})$ from $\left(\mathrm{AC}_{\circ}\right)$.

Proposition 5.6. If a function $x: I \rightarrow G$ is $\Xi_{j}$-maximal, then $\gamma=\psi\left(x j \ni_{R}\right)$ : $X \rightarrow Y$ is a quasi-choice of $\alpha$.

Proof. By Corollary $5.5 \gamma=\psi\left(x j \ni_{R}\right)$ is a univalent relation such that $\gamma \sqsubseteq \alpha$. We will show that $\gamma$ is a quasi-choice of $\alpha$. Assume that $\gamma^{\prime}: X \rightarrow Y$ is a univalent relation such that $\gamma \sqsubseteq \gamma^{\prime} \sqsubseteq \alpha$. Again by Corollary 5.5 there exists a function $y: I \rightarrow G$ such that $\gamma^{\prime}=\psi\left(y j \ni_{R}\right)$. Then it holds that

$$
x j \ni_{R}=\phi\left(\psi\left(x j \ni_{R}\right)\right)=\phi(\gamma) \sqsubseteq \phi\left(\gamma^{\prime}\right)=\phi\left(\psi\left(y j \ni_{R}\right)\right)=y j \ni_{R}
$$

and

$$
\begin{aligned}
y & \sqsubseteq x j \ni_{R} \triangleright \ni_{R}^{\sharp} j^{\sharp} & & \left\{x j \ni_{R} \sqsubseteq y j \ni_{R}\right\} \\
& =x \Xi_{j} & & \left\{x: \operatorname{tfn}, j \ni_{R} \triangleright \ni_{R}^{\sharp} j^{\sharp}=\Xi_{j}\right\} \\
& \sqsubseteq x, & & \left\{x: \Xi_{j} \text {-maximal }\right\}
\end{aligned}
$$

which implies $x=y$. Therefore one concludes

$$
\gamma=\psi\left(x j \ni_{R}\right)=\psi\left(y j \ni_{R}\right)=\gamma^{\prime},
$$

which proves that $\gamma$ is a quasi-choice of $\alpha$. 
The next proposition shows that a quasi-choice and a choice are equivalent under $(\mathrm{AC}-I)$.

Proposition 5.7. Suppose the restricted axiom of choice (AC-I). Every quasichoice of a relation $\alpha: X \rightarrow Y$ is a choice of $\alpha$.

Proof. Let $\gamma: X \rightarrow Y$ be a quasi-choice of $\alpha$. We will prove $\lfloor\gamma\rfloor=\lfloor\alpha\rfloor$, which is equivalent to $\alpha \sqcap\left(\gamma \nabla_{Y Y}\right)^{-}=0_{X Y}$. Set $\gamma^{\prime}=\alpha \sqcap\left(\gamma \nabla_{Y Y}\right)^{-}$. As $\gamma^{\prime}$ is included in $\alpha$, we obtain $\phi\left(\gamma^{\prime}\right): I \rightarrow R$ which bijectively corresponds to $\gamma^{\prime}$ by Proposition 5.3 (a). Also, by $(\mathrm{AC}-I)$, there exists a univalent relation $\nu: I \rightarrow R$ such that $\nu \sqsubseteq \phi\left(\gamma^{\prime}\right)$ and $\lfloor\nu\rfloor=\left\lfloor\phi\left(\gamma^{\prime}\right)\right\rfloor$. The univalency $\nu^{\sharp} \nu \sqsubseteq \operatorname{id}_{R}$ implies the condition $\nu^{\sharp} \nu \sqsubseteq p p^{\sharp} \Rightarrow \mathrm{id}_{R}$, and so $\psi(\nu)$ is a univalent relation such that $\psi(\nu) \sqsubseteq \alpha$ by Proposition $5.3(\mathrm{c})$. Since

$$
\begin{array}{rlrl}
\gamma \sqcap \psi(\nu) & \sqsubseteq \gamma \sqcap\left(\gamma \nabla_{Y Y}\right)^{-} & & \left\{\psi(\nu) \sqsubseteq \psi\left(\phi\left(\gamma^{\prime}\right)\right)=\gamma^{\prime}\right\} \\
& \sqsubseteq \alpha \nabla_{Y Y} \sqcap\left(\alpha \nabla_{Y Y}\right)^{-} & \left\{\operatorname{id}_{Y} \sqsubseteq \nabla_{Y Y}\right\} \\
& =0_{X Y}, & &
\end{array}
$$

$\gamma \sqcup \psi(\nu)$ is a univalent relation contained in $\alpha$. Hence by the maximality of $\gamma$, we have $\gamma \sqcup \psi(\nu)=\gamma$. Finally one can deduce $\gamma^{\prime}=0_{X Y}$ as follows:

$$
\begin{array}{ll}
\rightarrow \gamma=\gamma \sqcup \psi(\nu) & \\
\rightarrow \psi(\nu)=\gamma \sqcap \psi(\nu)=0_{X Y} & \\
\rightarrow \nu=0_{I R} & \\
\rightarrow\left\lfloor\phi\left(\gamma^{\prime}\right)\right\rfloor=\lfloor\nu\rfloor=0_{I I} & \\
\rightarrow \phi\left(\gamma^{\prime}\right)=0_{I R} & \\
\rightarrow \gamma^{\prime}=0_{X Y} . & \{\phi: \text { bijection }\}
\end{array}
$$

This completes the proof.

Finally we summarise the main results in the paper.

COROLlARY 5.8. The following three statements are equivalent.

(a) $\left(\mathrm{AC}_{*}\right)$,

(b) $(\mathrm{ZL}) \wedge(\mathrm{AC}-I)$,

(c) $\left(\mathrm{AC}_{\circ}\right) \wedge(\mathrm{AC}-I)$.

Proof. It is direct from the last propositions: (a) implies (b) by Theorem 5.2, (b) implies (c) by Proposition 5.6, and (c) implies (a) by Proposition 5.7.

\section{Kuratowski's lemma}

As an application of (ZL) we will give a relational proof of Kuratowski's lemma in Cantor categories satisfying the axiom of totality $\left(\operatorname{Tot}_{*}\right)$.

For a relation $\delta: X \rightarrow X$, a relation $\rho: V \rightarrow X$ is a $\delta$-clique if $\rho^{\sharp} \rho \sqsubseteq \delta$. Note that $\left(\xi \sqcup \xi^{\sharp}\right)$-cliques are $\xi$-chains and $\operatorname{id}_{X}$-cliques are univalent relations. 
Proposition 6.1. Let $j: D \rightarrow \wp(X)$ be an injection such that

$$
j^{\sharp} j=\operatorname{id}_{\wp(X)} \sqcap\left(\ni_{X} \triangleright \delta \triangleleft \ni_{X}^{\sharp}\right) \sqcap \nabla_{\wp(X) I} \sigma_{0}^{@} \Xi_{X},
$$

where $\delta: X \rightarrow X$ is a relation and $\sigma_{0}: I \rightarrow X$ is a $\delta$-clique.

(a) For all relations $\sigma: I \rightarrow X$ the following equivalences hold:

$$
\left\langle\sigma^{\sharp} \sigma \sqsubseteq \delta\right\rangle \wedge\left\langle\sigma_{0} \sqsubseteq \sigma\right\rangle \leftrightarrow\left\langle\sigma^{@ \sharp} \sigma^{@} \sqsubseteq j^{\sharp} j\right\rangle \leftrightarrow\left\langle\sigma^{@} j^{\sharp}: \operatorname{tfn}\right\rangle .
$$

(b) Suppose the axiom of totality $\left(\right.$ Tot $\left._{*}\right)$. The induced order $\Xi_{j}=j \Xi_{X} j^{\sharp}: D \rightarrow D$ is chain complete on $I$.

Proof. (a) The first equivalence follows from (1) and (2) below:

(1) $\sigma^{\sharp} \sigma \sqsubseteq \delta \leftrightarrow \sigma^{@ \sharp} \sigma^{@} \sqsubseteq \ni_{X} \triangleright \delta \triangleleft \ni_{X}^{\sharp}$ :

$$
\begin{aligned}
\sigma^{\sharp} \sigma \sqsubseteq \delta & \leftrightarrow \quad \ni_{X}^{\sharp} \sigma^{@ \sharp} \sigma^{@} \ni_{X} \sqsubseteq \delta \quad\left\{\sigma^{@} \ni_{X}=\sigma\right\} \\
& \leftrightarrow \sigma^{@ \sharp} \sigma^{@} \sqsubseteq \ni_{X} \triangleright \delta \triangleleft \ni_{X}^{\sharp} .
\end{aligned}
$$

(2) $\sigma_{0} \sqsubseteq \sigma \leftrightarrow \sigma^{@ \sharp} \sigma^{@} \sqsubseteq \nabla_{\wp(X) I} \sigma_{0}^{@} \Xi_{X}:$

$$
\begin{aligned}
\sigma_{0} \sqsubseteq \sigma & \leftrightarrow \sigma_{0} \sqsubseteq \sigma^{@} \ni_{X} & & \left\{\sigma=\sigma^{@} \ni_{X}\right\} \\
& \leftrightarrow \sigma_{0} \sqsubseteq \sigma^{@} \triangleright \ni_{X} & & \left\{\sigma^{@}: \text { function }\right\} \\
& \leftrightarrow \sigma^{@} \sqsubseteq \sigma_{0} \triangleright \ni_{X}^{\sharp} & & \{\text { Galois conn. }\} \\
& \leftrightarrow \sigma^{@} \sqsubseteq \sigma_{0}^{@} \Xi_{X} & & \left\{\sigma_{0}^{@}: \text { function }\right\} \\
& \leftrightarrow \sigma^{@ \sharp} \sigma^{@} \sqsubseteq \nabla_{\wp(X) I} \sigma_{0}^{@} \Xi_{X} . & & \{I \text { : unit }\}
\end{aligned}
$$

The second equivalence follows from (3) and (4) below: (3) $\sigma^{@ \sharp} \sigma^{@} \sqsubseteq j^{\sharp} j \rightarrow \sigma^{@} j^{\sharp}$ : function :

Assume $\sigma^{@} \sigma^{@} \sqsubseteq j^{\sharp} j$. Then

$$
\begin{array}{rlrl}
j \sigma^{@ \sharp} \sigma^{@} j^{\sharp} \sqsubseteq j j^{\sharp} j j^{\sharp} & \left\{\sigma^{@ \sharp} \sigma^{@} \sqsubseteq j^{\sharp} j\right\} \\
& =\operatorname{id}_{D} . & \left\{j j^{\sharp}=\mathrm{id}_{D}\right\} \\
\operatorname{id}_{I} \sqsubseteq \sigma^{@} \sigma^{@ \sharp} \sigma^{@} \sigma^{@ \sharp} & \left\{\sigma^{@}: \operatorname{tfn}\right\} \\
& \sqsubseteq \sigma^{@} j^{\sharp} j \sigma^{@ \sharp} . & \left\{\sigma^{\circledR} \sigma^{@} \sqsubseteq j^{\sharp} j\right\}
\end{array}
$$

Hence $\sigma^{@} j^{\sharp}$ is a function.

(4) $\sigma^{@} j^{\sharp}: \operatorname{tfn} \rightarrow \sigma^{@ \sharp} \sigma^{@} \sqsubseteq j^{\sharp} j:$

$$
\begin{aligned}
\sigma^{@} j^{\sharp}: \mathrm{tfn} & \rightarrow \sigma^{@} j^{\sharp} j=\sigma^{@} & \left\{\sigma^{@} j^{\sharp} j \sqsubseteq \sigma^{@}\right\} \\
& \rightarrow \sigma^{@} \sigma^{@}=\sigma^{@ \sharp} \sigma^{@} j^{\sharp} j & \\
& \rightarrow \sigma^{@ \sharp} \sigma^{@} \sqsubseteq j^{\sharp} j . & \left\{\sigma^{@}: \text { function }\right\}
\end{aligned}
$$

(b) We will show that for all $\Xi_{j}$-chains $\lambda: I \rightarrow D$ the $\operatorname{supremum} \sup \left(\lambda, \Xi_{j}\right)$ is total. Let's begin with the case that $\lambda=0_{I D}$. Note that $\sup \left(0_{I D}, \Xi_{j}\right)=\nabla_{I D} \triangleright \Xi_{j}^{\sharp}$ by $0_{I D} \triangleright \Xi_{j}=$ $\nabla_{I D}$, and $\sigma_{0}^{@} j^{\sharp} \sqsubseteq \nabla_{I D} \triangleright \Xi_{j}^{\sharp}=\sup \left(0_{I D}, \Xi_{j}\right)$ follows from

$$
\begin{aligned}
& \nabla_{I D}=\nabla_{I D} j j^{\sharp} j j^{\sharp} \quad\left\{j j^{\sharp}=\operatorname{id}_{D}\right\} \\
& \sqsubseteq \nabla_{I D} j \nabla_{\wp(X) I} \sigma_{0}^{@} \Xi_{X} j^{\sharp} \quad\left\{j^{\sharp} j \sqsubseteq \nabla_{\wp(X) I} \sigma_{0}^{@} \Xi_{X}\right\} \\
& \sqsubseteq \sigma_{0}^{\Theta} \Xi_{X} j^{\sharp} \quad\left\{\nabla_{I D} j \nabla_{\wp}(X) I \sqsubseteq \mathrm{id}_{I}\right\} \\
& =\sigma_{0}^{@} \triangleright \Xi_{X} j^{\sharp} \quad\left\{\sigma_{0}^{@}: \operatorname{tfn}\right\} \\
& \sqsubseteq \sigma_{0}^{@} j^{\sharp} j \triangleright \Xi_{X} j^{\sharp} \quad\left\{j^{\sharp} j \sqsubseteq \operatorname{id}_{\wp(X)}\right\} \\
& =\sigma_{0}^{@} j^{\sharp} \triangleright \Xi_{j} . \quad\{j \text { : function }\}
\end{aligned}
$$


Hence $\sup \left(0_{I D}, \Xi_{j}\right)$ is total, since $\sigma_{0}^{@} j^{\sharp}$ is a function by (a). Next the case that $\lambda \neq 0_{I D}$ will be shown. Note that $\lambda$ is total by the assumption ( $\left.\operatorname{Tot}_{*}\right)$. Then $\lambda j \ni_{X}$ is a $\delta$-clique such that $\sigma_{0} \sqsubseteq \lambda j \ni_{X}$ by

$$
\begin{aligned}
\left(\lambda j \ni_{X}\right)^{\sharp} \lambda j \ni_{X} & \sqsubseteq \ni_{X}^{\sharp} j^{\sharp} \Xi_{j} \Xi_{j}^{\sharp} j \ni_{X} & & \left\{\lambda^{\sharp} \lambda \sqsubseteq \Xi_{j} \sqcup \Xi_{j}^{\sharp} \sqsubseteq \Xi_{j} \Xi_{j}^{\sharp}\right\} \\
& \sqsubseteq \ni_{X}^{\sharp} j^{\sharp} j \ni_{X} & & \left\{\Xi_{j}^{\sharp} j \ni_{X} \sqsubseteq j \ni_{X}\right\} \\
& \sqsubseteq \delta & & \left\{\ni_{X}^{\sharp} j^{\sharp} j \ni_{X} \sqsubseteq \delta\right\}
\end{aligned}
$$

and

$$
\begin{array}{rlll}
\sigma_{0} & \sqsubseteq\left(\sigma_{0} \triangleright \ni_{X}^{\sharp}\right) \triangleright \ni_{X} & & \\
& \sqsubseteq\left(\lambda j \nabla_{\wp(X) I}\left(\sigma_{0} \triangleright \ni_{X}^{\sharp}\right)\right) \triangleright \ni_{X} & & \left\{\lambda j \nabla_{\wp(X) I} \sqsubseteq \mathrm{id}_{I}\right\} \\
& \sqsubseteq \lambda j j^{\sharp} \triangleright \ni_{X} & & \left\{j^{\sharp} \sqsubseteq \nabla_{\wp(X) I}\left(\sigma_{0} \triangleright \ni_{X}^{\sharp}\right)\right\} \\
& =\lambda j \triangleright \ni_{X} & & \left\{j j^{\sharp}=\operatorname{id}_{D}\right\} \\
& \sqsubseteq \lambda j \ni_{X} . & & \{\lambda, j: \text { total }\}
\end{array}
$$

Hence, by (a), $\left(\lambda j \ni_{X}\right)^{@} j^{\sharp}$ is a function. Moreover, by

$$
\begin{aligned}
\left(\lambda j \ni_{X}\right)^{@} j^{\sharp} & =\sup \left(\lambda j, \Xi_{X}\right) j^{\sharp} & & \left\{4.4(\mathrm{c})\left(\lambda j \ni_{X}\right)^{@}=\sup \left(\lambda j, \Xi_{X}\right)\right\} \\
& \sqsubseteq \sup \left(\lambda, \Xi_{j}\right) . & & \{2.7(\mathrm{f})\}
\end{aligned}
$$

$\sup \left(\lambda, \Xi_{j}\right)$ is total.

Kuratowski's lemma in Cantor categories with $\left(\right.$ Tot $\left._{*}\right)$ is stated as follows.

Corollary 6.2. For all relations $\delta: X \rightarrow X$ every $\delta$-clique $\sigma_{0}: I \rightarrow X$ is contained in a maximal $\delta$-clique.

Proof. By the axiom of rationality (Rat) there exists an injection $j: D \rightarrow \wp(X)$ such that $j^{\sharp} j=\operatorname{id}_{\wp(X)} \sqcap\left(\ni_{X} \triangleright \delta \triangleleft \ni_{X}^{\sharp}\right) \sqcap \nabla_{\wp(X) I} \sigma_{0}^{@} \Xi_{X}$. The induced order $\Xi_{j}$ is chain complete on $I$ by Proposition 6.1 (b), and so it is inductive. Hence by Zorn's lemma there exists a $\Xi_{j}$-maximal function $x: I \rightarrow D$. It follows from (1) and (2) below that $x j \ni_{X}$ is a maximal $\delta$-clique such that $\sigma_{0} \sqsubseteq x j \ni_{X}$.

(1) $x j \ni_{X}: I \rightarrow X$ is a $\delta$-clique such that $\sigma_{0} \sqsubseteq x j \ni_{X}$.

First recall $\left(x j \ni_{X}\right)^{@}=x j$ and $\left(x j \ni_{X}\right)^{@} j^{\sharp}=x j j^{\sharp}=x$ is a function. By Proposition 6.1

(a) $x j \ni_{X}$ is a $\delta$-clique such that $\sigma_{0} \sqsubseteq x j \ni_{X}$.

(2) If $\sigma: I \rightarrow X$ is a $\delta$-clique such that $x j \ni_{X} \sqsubseteq \sigma$, then $\sigma=x j \ni_{X}$.

By (1) $\sigma$ is a $\delta$-clique such that $\sigma_{0} \sqsubseteq \sigma$, and $\sigma^{@} j^{\sharp}$ is a function by Proposition 6.1 (a). Hence

$$
\begin{array}{rlrl}
\sigma & =\sigma^{@} \ni_{X} & & \\
& =\sigma^{@} j^{\sharp} j \ni_{X} & & \left\{\sigma^{@} j^{\sharp}: \mathrm{tfn}\right\} \\
& \sqsubseteq\left(\sigma \triangleright \ni_{X}^{\sharp}\right) j^{\sharp} j \ni_{X} & & \left\{\sigma^{@} \sqsubseteq \sigma \triangleright \ni_{X}^{\sharp}\right\} \\
& \sqsubseteq\left(x j \ni_{X} \triangleright \ni_{X}^{\sharp}\right) j^{\sharp} j \ni_{X} & & \left\{x j \ni_{X} \sqsubseteq \sigma\right\} \\
& =x j \Xi_{X} j j \ni_{X} & & \{x j: \mathrm{tfn}\} \\
& =x \Xi_{j} j \ni_{X} & & \left\{j \Xi_{X} j^{\sharp}=\Xi_{j}\right\} \\
\sqsubseteq x j \ni_{X}, & & \left\{x: \Xi_{j} \text {-maximal }\right\}
\end{array}
$$

which proves that $\sigma=x j \ni_{X}$. 


\section{Conclusion}

We gave a relational proof in Cantor categories satisfying $(\mathrm{Ba})$ to the known equivalence of axiom of choice and Zorn's lemma, based on a classical proof [Dugundji (1996)]. The result is illustrated as follows.

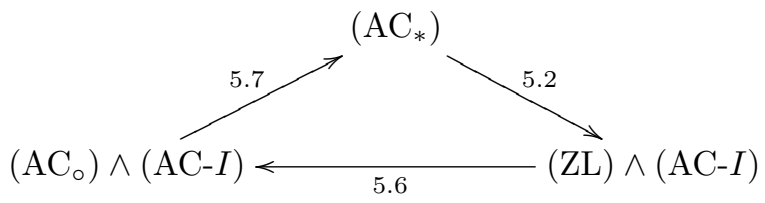

A relational proof of Kuratowski's lemma in Cantor categories satisfying (Ba) and ( Tot $\left._{*}\right)$ was also given as an application of (ZL). In set theory the equivalence of (AC) and (ZL) is recently deduced by using theory of ordinal numbers, and so the relational study of ordinal numbers in Dedekind categories may be an interesting future work.

\section{Acknowledgement}

The presentation of this article has benefited from the anonymous reviewer's suggestions. This work was partly supported by JSPS KAKENHI Grant Number JP25330016, JP16K21557.

\section{References}

Rudolf Berghammer and Gunther Schmidt (1991). The RELVIEW-System. Lecture Notes in Computer Science 480, 535-536 (website of the system: https://www . informatik. uni-kiel.de/ progsys/relview/)

James Dugundji (1996). Topology. Allyn and Bacon

Peter Freyd and Andre Scedrov (1990). Categories, Allegories. North-Holland

Hitoshi Furusawa and Yasuo Kawahara (2015). Point Axioms and Related Conditions in Dedekind Categories. Journal of Logic and Algebraic Methods in Programming 84(3), 359-376

Joseph Goguen (1967). L-Fuzzy Sets. Journal of Mathematical Analysis and Applications 18(1), 145-174

Peter Höfner and Georg Struth (2008). On Automating the Calculus of Relations. Lecture Notes in Computer Science 5195, 50-66

Wolfram Kahl (2014). RATH-Agda. Relation-Algebraic Theories in Agda: http:// relmics.mcmaster.ca/RATH-Agda/

Dylan Killingbeck (2015). A Generic Matrix Manipulator. Proceedings of the Student Track of RAMiCS 2015, 61-69

Saunders Mac Lane (1971). Categories for the Working Mathematician. Springer

Jean-Pierre Olivier and Dany Serrato (1980). Catégories de Dedekind. Morphismes dans les Catégories de Schröder. Comptes Rendus Hebdomadaires des Séances de l'Académie des Sciences 290(20), A939-A941 
Damien Pous (2012). Relation Algebra and KAT in Coq: http://perso.ens-lyon. fr/damien.pous/ra/

Herman Rubin and Jean E. Rubin (1963). Equivalents of the Axiom of Choice. NorthHolland

Gunther Schmidt and Thomas Ströhlein (1993). Relations and Graphs. Discrete Mathematics for Computer Scientists. Springer

Georg Struth and Tjark Weber (2014). Relation Algebra. Archive of Formal Proofs 2014: http://www.isa-afp.org/entries/Relation_Algebra.shtml

Alfred Tarski (1941). On the Calculus of Relations. Journal of Symbolic Logic 6, 73-89

Ernst Zermelo (1904). Beweis, dass jede Menge wohlgeordnet werden kann. Mathematische Annalen 59(4), 514-516

Max Zorn (1935). A Remark on Method in Transfinite Algebra, Bulletin of the American Mathematical Society 41(10), 667-670

Received July 25, 2016

Revised February 17, 2017 